\title{
Transversal Dirac Families in Riemannian Foliations
}

\author{
J. F. Glazebrook ${ }^{1}$ and F. W. Kamber ${ }^{2, \star}$ \\ 1 Department of Mathematics, Eastern Illinois University, Charleston, IL 61920, USA \\ 2 Department of Mathematics, University of Illinois, 1409 West Green, Urbana, IL 61801, USA
}

Received November 13, 1989; in revised form September 4, 1990

\begin{abstract}
We describe a family of differential operators parametrized by the transversal vector potentials of a Riemannian foliation relative to the Clifford algebra of the foliation. This family is non-elliptic but in certain ways behaves like a standard Dirac family in the absolute case as a result of its elliptic-like regularity properties. The analytic and topological indices of this family are defined as elements of K-theory in the parameter space. We indicate how the cohomology of the parameter space is described via suitable maps to Fredholm operators. We outline the proof of a theorem of Vafa-Witten type on uniform bounds for the eigenvalues of this family using a spectral flow argument. A determinant operator is also defined with the appropriate zeta function regularization dependent on the codimension of the foliation. With respect to a generalized coupled DiracYang-Mills system, we indicate how chiral anomalies are located relative to the foliation.
\end{abstract}

\section{Introduction}

This paper provides a setting for the study of a coupled Dirac-Yang-Mills theory in the presence of a Riemannian foliation $\mathscr{F}$. The latter may be regarded in essence as a generalized dynamical system in which a one-dimensional foliation is simply called a flow. Among a number of possible applications suggested in this paper, we mention at this stage a particular example which is well known and which may serve as a partial motivation for what follows: The pure Yang-Mills equations on $\mathbb{R}^{4}$ may be dimensionally reduced by requiring translation invariance in one direction, thus arriving at a Higgs system in $\mathbb{R}^{3}$ yielding magnetic monopole equations $[\mathrm{A}-\mathrm{H}][\mathrm{J}-\mathrm{T}]$. One may see the generalization of translation invariance in the above example as invariance under the flows in the leaf direction of a foliation $\mathscr{F}$. In the context of vector potentials this leads to the notion of a basic connection

\footnotetext{
* Work supported in part by a grant from the National Science Foundation
} 
form with respect to $\mathscr{F}$. It is clear that the choice of vector potentials must in some way reflect the invariance under these flows. On the other hand, the leaf space of the foliation is in general highly singular, but still has some interesting structure (Molino theory [Mo]). In the case where this happens to be an orbifold, the study of anomalies relative to the heterotic string has been described by a number of authors (see e.g. $[\mathrm{F}-\mathrm{V}]$ ). From an analytic stance, the resulting spectral analysis on the singular leaf space is a deep topic which is now receiving much attention and new index type theorems are expected (see e.g. the survey article by Brüning $[\mathrm{Br}]$ ). It would be reasonable then to suggest a fruitful interaction with gauge and string theories within the context of foliation theory.

Modulo the technical details, the overall theme of the paper is to consider a new class of anomalies relative to a non-elliptic family of geometric Dirac operators defined by the transversal structure of the foliation and parametrized by an orbit space of basic vector potentials. One interesting offshoot of our work is a generalization of some results of Vafa-Witten $[\mathrm{V}-\mathrm{W}]$ on fermion inequalities, although our approach is fundamentally topological. For the best part, the anomalies we consider are chiral and our results may have a number of applications to quantum chromodynamics besides the aforesaid Yang-Mills-Higgs system. These may be seen as our motivating examples.

One commences with a principal $G$-bundle $P \rightarrow M$, where $M$ is a (compact) manifold carrying a Riemannian foliation $\mathscr{F}$ of codimension $q$ which lifts to a foliated structure of $P$. Basic connections on $P$ have been studied by a number of authors [Bo], [K-T1], [Mo] and they figure prominently in the theory of characteristic classes of foliations as well as in equivariant cohomology theory [A-B2]. The relevant spin structures are inherent to the transversal geometry of the foliation and not necessarily to $M$ itself. We introduce a basic Dirac operator $\not D_{b}$ operating on the holonomy invariant (fermionic) sections of a twisted foliated spin bundle on $M$. Generalized Dirac operators, in the absolute case (i.e. $q=\operatorname{dim} M$ ) were considered in the work of Gromov-Lawson [G-L 1,2] and more recently by J. Roe [Ro2,3]. These operators, in our case, are non-elliptic for general codimension, but as a consequence of their restriction to holonomy invariant sections, their spectral properties are fairly well tempered and remarkably, each operator does exhibit elliptic-like regularity. Thus we can apply $K$-theoretic methods to study their null-spaces (zero-frequency modes). For $q$ even, we find chiral anomalies with corresponding determinants regularized by the basic zeta-function technique. Now the significant difference here with the absolute case is that the zeta function regularity genuinely reflects the structure of the foliation. In the absolute case, with $M$ an even dimensional spin manifold, the situation described by Atiyah-Singer [A-S4] and Singer [Si] is recovered (the basic Dirac family now coincides with the standard elliptic one).

Our results in part are derived in an analogous fashion, but a number of non-trivial modifications are required. On the cohomological level, our relative anomalies have the analogous interpretation as in e.g. [B-C-R-S]. However, the choice of taking basic connections on the principal bundle $P$ will lead to further characteristic classes determined by the foliation [K-T1]. We will report on this aspect in a follow up to this paper [G1-K2].

Before outlining the content of the paper, we will establish the relevant definitions in the context of foliation theory. We will assume that the reader has 
some acquaintance with the theory of Riemannian foliations; suitable references are e.g. $[\mathrm{K}-\mathrm{T}, 1,4],[\mathrm{Mo}],[\mathrm{Re} 1],[\mathrm{Re} 2]$. The discussion immediately following is based on recent work of the second named author and J. Brüning [B-K1], [B-K2].

Let $M$ be an oriented compact Riemannian manifold of dimension $m$ and $\left(M, \mathscr{F}, g_{M}\right)$ an oriented Riemannian foliation on $M$ of codimension $q$ with bundle-like metric $g_{M}$.

We recall the exact sequence

$$
0 \rightarrow L(\mathscr{F}) \rightarrow T M \stackrel{\pi}{\rightarrow} Q \rightarrow 0
$$

determined by the tangent bundle and the normal bundle of $\mathscr{F}$. Let $E$ be a complex Hermitian foliated bundle over $M$ which is a Clifford module over $\mathrm{Cl}(Q)$, the transversal Clifford algebra of $\mathscr{F}$ and let $\mathrm{cl}=: C^{\infty}(Q \otimes E) \rightarrow C^{\infty}(E)$ denote multiplication. Taking $\hat{\pi}$ to denote the projection

$$
\hat{\pi}: C^{\infty}\left(T^{*} M \otimes E\right) \rightarrow C^{\infty}\left(Q^{*} \otimes E\right) \cong C^{\infty}(Q \otimes E)
$$

we define the transversal Dirac operator $\not_{\mathrm{tr}}^{\prime}$ as a generalized Dirac operator in the sense of $[\mathrm{G}-\mathrm{L} 1,2],[\mathrm{Ro1}, 2,3]$ by

$$
\not D_{\mathrm{tr}}^{\prime}=\operatorname{cl} \circ \hat{\pi}^{\circ} \circ \nabla^{E},
$$

where $\nabla=\nabla^{E}$ denotes covariant differentiation with respect to the metric connection on $E$. If $\left\{E_{\alpha}\right\}_{\alpha=1, \ldots, q}$ is taken to be a local orthonormal projectable frame in $Q$, then

$$
\not D_{\mathrm{tr}}^{\prime}=\sum_{\alpha} E_{\alpha} \cdot \nabla_{E_{\alpha}}
$$

Taking $\chi_{F}$ to denote the characteristic volume form along the foliation, then modulo $\mathscr{F}$-trivial forms, Rummler's formula $[\mathrm{Ru}]$ states that

$$
d \chi_{\mathscr{F}}+\kappa \wedge \chi_{\mathscr{F}}=0,
$$

where $\kappa \in C^{\infty}\left(Q^{*}\right)$ denotes the mean curvature of $\left(M, \mathscr{F}, g_{M}\right)$. In [B-K2] it was shown that the formal adjoint $\left(\mathbb{D}_{\mathrm{tr}}^{\prime}\right)^{*}$ is given by $\left(\not_{\mathrm{tr}}^{\prime}\right)^{*}=\not_{\mathrm{tr}}^{\prime}-\kappa \cdot$ and that therefore

$$
D_{\mathrm{tr}}=D_{\mathrm{tr}}^{\prime}-\frac{1}{2} \kappa
$$

is a symmetric, transversally elliptic differential operator, with symbol $\sigma$ satisfying $\sigma(x, \xi)=\xi \cdot$ for $\xi \in Q_{x}^{*}$ and $\sigma(x, \xi)=0$ for $\xi \in L_{x}^{*}$. Perhaps we should remark that the mean curvature term seems to appear already in Witten's paper [W]. We define the subspace $\Gamma_{b}(E)$ of basic or holonomy invariant sections of $E$ by

$$
\Gamma_{b}(E)=\left\{s \in C^{\infty}(E): \nabla_{X} s=0, \quad X \in C^{\infty}(L(\mathscr{F}))\right\} ;
$$

observing that for the forms $E=\Lambda Q^{*} \otimes \mathbb{C}$ on the normal bundle we have

$$
\Gamma_{b}(E)=\Omega_{b}(\mathscr{F}),
$$

where $\Omega_{b}(\mathscr{F})$ are the usual $(\mathbb{C}$-valued) basic forms of $(M, \mathscr{F})$. From $(1.6)$ we see that $D_{\mathrm{tr}}$ leaves $\Gamma_{b}(E)$ invariant if and only if the foliation $\left(M, \mathscr{F}, g_{M}\right)$ is isoparametric, i.e. $\kappa \in \Omega_{b}^{1}(\mathscr{F})$, in which case $\kappa$ is closed [K-T5]. Let

$$
\not D_{b}=\not_{\mathrm{tr}} \mid \Gamma_{b}(E): \Gamma_{b}(E) \rightarrow \Gamma_{b}(E) ;
$$


we refer to this operator $\not_{b}$ as the basic Dirac operator on (smooth) basic sections $\Gamma_{b}(E)$.

In [B-K2] certain vanishing theorems and index formulae were established for the operators $D_{\mathrm{tr}}, D_{b}$ and their powers. Indeed these operators are essentially self-adjoint with $\not_{b}$ and $\not_{b}^{2}$ each possessing a discrete spectrum. The special case of the basic Laplace operator was studied in [K-T3] (compare also [A1], [Co], $[\mathrm{EK}],[\mathrm{EK}-\mathrm{H}])$.

We now outline the rest of the paper. In Sect. 2 we discuss some properties of $\not D_{b}$ with a view to the Bochner-Lichnerowicz technique. Then in Sect. 3 we introduce the basic vector potentials relative to the notion of infinitesimal flow (Lie derivative) in the leaf directions. We allow these to vary in a moduli space $Y$ which is a quotient by the gauge group of the foliation. Elaborating on our earlier remark, we briefly describe a reduced YM system relative to a Killing vector field flow on a 4-manifold. The topological and analytic indices are defined in the $K$-theory of $Y$. Since our operators possess elliptic-like regularity, but are not elliptic themselves, a certain amount of reworking drawn from [A-S2] is necessary. This is dealt with in Sects. 4 and 5. Maps from $Y$ to Fredholm operators (for both parities of the codimension) are treated in Sect. 6 leading to the notion of $\mathscr{F}$-relative anomalies. Here the foliated bundle appears as a functorial variable. Section 7 is devoted to a generalization of the results of Vafa and Witten [V-W] by means of a spectral flow approach. We state the analogous fermion inequalities in the transversal direction. We exemplify matters by indicating that for codimension $q=4$, the analogue of the absence of a mass gap occurs under the appropriate conditions. In Sect. 8 we introduce the basic zeta function which (for $q$ even) allows us to regularize determinants as in the absolute case. Anomalies can then be located and are interpreted as an obstruction to a gauge-invariant determinant for the basic Dirac family.

\section{Properties of the Basic Dirac Operator}

The following properties of the operators $D_{\mathrm{tr}}$ and $D_{b}$ were established in [B-K2]:

(i) On $\mathrm{C}^{\infty}(E)$, we have the Bochner-Weitzenböck type formula

$$
\not D_{\mathrm{tr}}^{2}=\nabla_{\mathrm{tr}}^{*} \nabla_{\mathrm{tr}}+\mathscr{R}_{\nabla}+\mathscr{K}_{\nabla}
$$

where

$$
\nabla_{\mathrm{tr}}^{*}=\Sigma_{\alpha} i\left(E_{\alpha}\right) \nabla_{E_{\alpha}}^{-\kappa}
$$

and

$$
\mathscr{R}_{\nabla}(s)=\Sigma_{\alpha<\beta} E_{\alpha} \cdot E_{\beta} \cdot R_{\nabla}\left(E_{\alpha}, E_{\beta}\right)(s), \quad \mathscr{K}_{\nabla}=\frac{1}{2}\left\{-\delta \kappa+\frac{1}{2}|\kappa|^{2}\right\} \cdot,
$$

with $\mathscr{R}_{\nabla}, \mathscr{K}_{\nabla}$ pointwise symmetric.

(ii) On $\Gamma_{b}(E)$, we have

$$
\not D_{b}^{2}=\Delta \mid \Gamma_{b}(E),
$$

where $\Delta=\nabla^{*} \nabla+\mathscr{R}_{\nabla}+\mathscr{K}_{\nabla}$ is a strongly elliptic, symmetric operator of Laplace type. 
The restriction property (2.3) may be used to prove vanishing theorems for $\operatorname{ker}\left(D_{b}\right)$, provided one is able to control the divergence term $\delta \kappa$ in the above expression for $\mathscr{K}_{\nabla}$. The easiest condition to impose is that $\delta \kappa=0$, i.e. that $\kappa$, being already closed, is a harmonic 1 -form. We then have $\mathscr{K}_{\nabla}=\frac{1}{4}|\kappa|^{2}$ and the resulting local equation

$$
g_{E}\left(D_{b}^{2} s, s\right)=\frac{1}{2} \Delta|s|^{2}+|\nabla s|^{2}+g_{E}\left(\mathscr{R}_{\nabla}(s), s\right)+\frac{1}{4}|\kappa|^{2}|s|^{2}
$$

implies transversal vanishing theorems for $\operatorname{ker}\left(\not_{b}\right)$ by the usual BochnerLichnerowicz argument, provided $\mathscr{R}_{\nabla} \geqq 0$ and $\mathscr{R}_{\nabla}$ is positive at least at one point $x_{0} \in M[\mathrm{G}-\mathrm{L} 1,2]$, [Li]. From our point of veiw, however, the main consequence of the restriction property (2.3) is given below.

(iii) The operators $D_{\mathrm{tr}}, D_{b}$ and their powers are essentially self-adjoint on $L^{2}(E)$, respectively $L^{2}\left(\Gamma_{b}(E)\right)$, with $\not_{b}$ and $\not_{b}^{2}$ having discrete spectrum (i.e. $\operatorname{Spec}_{\text {ess }}\left(D_{b}\right)=\emptyset$ ). The absolute growth of the eigenvalues of $D_{b}$ is regulated by $\not_{b}^{2}$. The eigenspace decompositions of $\Delta$ and $\not_{b}^{2}$ are compatible under the following inclusions and projections

$$
\begin{aligned}
L^{2}\left(\mathrm{C}^{\infty}(E)\right) & =\coprod_{\lambda} E_{\lambda}(\Delta) \supset C^{\infty}(E) \\
\Pi \downarrow \bigcup_{\downarrow} \Pi \uparrow & \Pi{ }^{2}\left(\Gamma_{b}(E)\right)=\coprod_{\lambda} E_{\lambda}\left(\not D_{b}^{2}\right) \supset \Gamma_{b}(E)
\end{aligned}
$$

where $\Pi$ denotes orthogonal projection, and (2.5) is compatible with all Sobolev space completions $H_{s}(E)$ (respectively $H_{s}\left(\Gamma_{b}(E)\right.$ ).

From the above considerations we can deduce the elliptic-like regularity for $D_{b}$ :

Lemma 2.1. Let $\not_{b} v=s$ with $s \in \Gamma_{b}(E), v \in H_{s}\left(\Gamma_{b}(E)\right)$, then $v \in \Gamma_{b}(E)$.

In fact, $\not_{b}^{2}$ satisfies full elliptic regularity as a consequence of (iii) above and therefore $\not_{b}^{2} v=\not_{b} s \in \Gamma_{b}(E)$ implies $v \in \Gamma_{b}(E)$.

In the case where $q$ is even, the operator $D_{b}$ splits as

$$
\not_{b}=\left[\begin{array}{cc}
0 & \not_{b}^{-} \\
\not_{b}^{+} & 0
\end{array}\right] \text { and } \not_{b}^{2}=\left[\begin{array}{cc}
\not_{b}^{-} \not_{b}^{+} & 0 \\
0 & \not_{b}^{+} \not D_{b}^{-}
\end{array}\right] \text {, }
$$

i.e. $E=E^{+} \oplus E^{-}, \not_{b}^{+}: \Gamma_{b}\left(E^{+}\right) \rightarrow \Gamma_{b}\left(E^{-}\right), \not_{b}^{-}: \Gamma_{b}\left(E^{-}\right) \rightarrow \Gamma_{b}\left(E^{+}\right)$, with $\not D_{b}^{-}=\left(\not D_{b}^{+}\right)^{*}$

The following Lemma is immediate from Lemma 2.1 .

Lemma 2.2. Let $\not_{b}^{+} u=v$ with $v \in \Gamma_{b}\left(E^{-}\right), u \in H_{s}\left(\Gamma_{b}\left(E^{+}\right)\right)$, then $u \in \Gamma_{b}\left(E^{+}\right)$.

Theorem 2.3. [B-K2].

(i) The basic heat-operator $e^{-t \Phi_{b}^{2}}$ is a smoothing operator of trace class and kernel $K_{b, t} \in \Gamma_{b}\left(E^{*} \bowtie E, \mathscr{F} \times \mathscr{F}\right)$, for $t \geqq 0$;

(ii) $\left(e^{-t p_{b}^{2}}\right) u \rightarrow \Pi(u)$ in the $C^{\infty}$-topology for $t \uparrow \infty$ and $u \in L^{2}\left(\Gamma_{b}(E)\right)$, where $\Pi: L^{2}\left(\Gamma_{b}(E)\right) \rightarrow \operatorname{ker}\left(\not_{b}^{2}\right)$ is the orthogonal projection;

(iii) $\operatorname{Tr}_{s}\left(e^{-t \phi_{b}^{2}}\right)$ is independent of $t>0$, where $\operatorname{Tr}_{s}$ denotes supertrace;

(iv) The index of $D_{b}^{+}$is given by the following formula:

$$
\operatorname{Ind}\left(\not_{b}^{+}\right) \equiv \operatorname{ker}\left(\not_{b}^{+}\right)-\operatorname{ker}\left(\not_{b}^{-}\right)=\lim _{t \downarrow 0} \operatorname{Tr}_{s}\left(e^{-i \not_{b}^{2}}\right)
$$


Indeed, this follows as in $[\mathrm{A}-\mathrm{B}-\mathrm{P}]$, using the previous results. We have $\operatorname{ker}\left(\not_{b}^{+}\right)=\operatorname{ker}\left(\not_{b}^{-} \not_{b}^{+}\right), \operatorname{ker}\left(\not_{b}^{-}\right)=\operatorname{ker}\left(\not_{b}^{+} \not_{b}^{-}\right)$and $\not_{b}^{ \pm}$induce inverse isomorphisms of the eigenspaces $E_{\lambda}\left(\not_{b}^{-} \not D_{b}^{+}\right)$and $E_{\lambda}\left(\not_{b}^{+} \not D_{b}^{-}\right)$for $\lambda>0$.

\section{Remarks 2.4.}

(i) It follows from Lemma 2.2 that $\not_{b}^{+}$has closed range in $L^{2}\left(\Gamma_{b}(E)\right)$ and that therefore the operators of the type $\not_{b}^{+}$are Fredholm in the unbounded sense. But then, these operators may be turned into bounded Fredholm operators by appropriately altering norms in the Sobolev spaces.

(ii) Alternatively, the smoothness of the projection in (2.5) implies that the resolvent $R\left(\not_{b}^{2}, \zeta\right)=\left(\not_{b}^{2}-\zeta\right)^{-1}, \zeta \notin \operatorname{Spec}\left(\not_{b}^{2}\right), \zeta \neq 0$, is the restriction of the corresponding resolvent of the strongly elliptic operator $\Delta$ to $L^{2}\left(\Gamma_{b}(E)\right)$. Hence it is compact, symmetric and $\Psi_{\zeta}=I+\zeta R\left(\not_{b}^{2}, \zeta\right)$ is a bounded symmetric Fredholm operator having the same kernel and range as $D_{b}$ and whose spectrum is given by

$$
\operatorname{Spec}\left(\Psi_{\zeta}\right)=\left\{\mu=\lambda / \lambda-\zeta: \lambda \in \operatorname{Spec}\left(\not_{b}^{2}\right)\right\}
$$

We tacitly assume that $\Gamma_{b}(E)$ is infinite dimensional, hence $L^{2}\left(\Gamma_{b}(E)\right)$ is isomorphic to a separable complex Hilbert space.

\section{Indexing a Family of the Operators $\not_{b}$}

In what follows we shall assume that $Q$ is endowed with a $\operatorname{Spin}(q)$ structure $\mathbb{Z}_{2} \rightarrow F_{\text {spin }}(Q) \rightarrow F_{\text {So }}(Q)$ and take

$$
E=S \otimes V
$$

where $S=F_{\text {Spin }}(Q) \times{ }_{\operatorname{Spin}(q)} \Delta_{s}$ denotes the spin bundle associated to $Q, \Delta_{s}$ the spin representation and $V$ is a complex coefficient bundle. Indeed, we view $V$ as the vector bundle associated to a foliated principal $G$-bundle $P \rightarrow M[\mathrm{~K}-\mathrm{T} 1]$. For instance, we could take the corresponding $G$-bundle of a Riemannian $G$-foliation, where $P$ is the $G$-reduction of the principal frame bundle $F_{S o}(Q)$.

Throughout this paper we assume that $G$ denotes a compact, connected Lie group. Let $\rho: G \rightarrow S U(r)$ be a representation of $G$ on $\mathbb{C}^{r}$ and let $V=P \times{ }_{\rho} \mathbb{C}^{r}$ be the resulting complex vector bundle endowed with a Hermitian structure. We call the resulting bundle $E$ in (3.1) a foliated twisted spin bundle.

Let $\mathscr{A}$ denote the convex space of connections in $P$. We consider the restricted gauge group $\mathscr{G}=\mathscr{G}_{u_{0}} \subset \operatorname{ker}\left(C^{\infty}(\right.$ Aut $\left.P) \rightarrow \operatorname{Diff}(M)\right)$ of gauge transformations which fix a point $u_{0} \in P$ and denote the (free) action of $\mathscr{G}$ on $\mathscr{A}$ by $\phi \cdot A[\mathrm{~A}-\mathrm{J}]$, [A-S4], [Si]. We observe that $\phi \in \mathscr{G}$ is determined by a smooth mapping $\psi: P \rightarrow G$ via $\phi(u)=R_{\psi(u)}(u)=u \psi(u)$, satisfying $\psi(u g)=g^{-1} \psi(u) g, u \in P, g \in G[\mathrm{~A}-\mathrm{B} 1]$. Hence the differential $\psi_{*}: T P \rightarrow \mathfrak{g}$ satisfies $\psi_{*} \circ R_{g^{*}}=\operatorname{Ad}(g)^{-1} \cdot \psi_{*}$. We have

$$
\phi_{*}(X)=R_{\psi(u)^{*}}(X)+\psi_{*}(X)^{*}, \quad X \in T_{u} P,
$$

where $\xi^{*}$ is the vertical vectorfield determined by $\xi \in \mathfrak{g}$. It follows immediately that 
the action of $\mathscr{G}$ on $\mathscr{A}$ can be expressed by the formula

$$
\begin{aligned}
(\phi \cdot A)_{u} & =\left(\phi^{*}(A)\right)_{u} \\
& =\left(R_{\psi(u)}^{*} A\right)_{u}+\psi_{*, u} \\
& =\operatorname{Ad}(\psi(u))^{-1} \cdot A_{u}+\psi_{*, u}, \quad u \in P .
\end{aligned}
$$

Now the foliated structure on $P$ determines a canonical lift of vectorfields $X \in L(\mathscr{F})$ to $G$-invariant projectable vectorfields $\tilde{X} \in L(\tilde{\mathscr{F}})\left(\right.$ i.e. $R_{g^{\star}}(\tilde{X})=\tilde{X}$ for $\left.g \in G\right)$, where $\tilde{\mathscr{F}}$ denotes the $G$-invariant foliation on $P$ [K-T1]. A connection $A \in \mathscr{A}$ is called adapted to $\tilde{\mathscr{F}}$ if $i_{\tilde{X}} A=0$ and basic if, in addition, $L_{\tilde{X}} A=i_{\tilde{X}} d A=0$ for all vectorfields of the form $\tilde{X} \in L(\tilde{\mathscr{F}})$ (these are the transversally projectable connections of [Mo], cf. also [Bo]). We denote by $\mathscr{A}_{b} \subset \mathscr{A}$, the convex subset of basic connections in $P$. As $\mathscr{G}$ induces the identity on $M$ and the lifts $\tilde{X}$ are unique, it follows from (3.2) that the group $\mathscr{G}(\tilde{\mathscr{F}})=\mathscr{G} \cap \operatorname{Aut}(P, \tilde{\mathscr{F}})$ of gauge transformations preserving $\tilde{\mathscr{F}}$ (i.e. $\left.\phi_{*}(\tilde{X})=\tilde{X}\right)$ is given exactly by those $\phi \in \mathscr{G}$, for which $\psi_{*} \mid L(\tilde{\mathscr{F}})=0$, i.e. $\psi$ is constant on the leaves of $\tilde{\mathscr{F}}$. We call $\mathscr{G}(\tilde{\mathscr{F}})$ the basic gauge group of $(P, \tilde{\mathscr{F}})$. The $G$-invariance of the vectorfields $\tilde{X}$ implies that the $G$-action $R_{g}^{*}$ and the Lie derivatives $L_{\tilde{X}}$ commute on $\mathscr{A}$; (3.2) and (3.3) then imply that $\mathscr{A}_{b}$ is invariant under $\phi \in \mathscr{G}(\tilde{\mathscr{F}})$. We summarize this in the following proposition.

\section{Proposition 3.1}

(i) $\mathscr{G}(\tilde{\mathscr{F}})$ consists of those gauge transformations in $\mathscr{G}$ for which $\psi_{*} \mid L(\tilde{\mathscr{F}})=0$ for all $\tilde{X} \in L(\tilde{\mathscr{F}})$;

(ii) The convex subspace of basic connections $\mathscr{A}_{b} \subset \mathscr{A}$ is invariant under the action of $\mathscr{G}(\tilde{\mathscr{F}})$ and $\mathscr{G}(\tilde{\mathscr{F}})$ acts freelly on $\mathscr{A}_{b}$.

Remark 3.2. For a basic connection $A$, the curvature from $F_{A}$ is a basic form, that is $i_{\tilde{X}} F_{A}=0$ and $L_{\tilde{X}} F_{A}=0$.

Let $\phi \in \mathscr{G}(\tilde{\mathscr{F}})$ and take $\tilde{\phi}$ to denote its extension via $\rho$, as a basic element $\tilde{\phi} \in C^{\infty}(\operatorname{Aut}(V))$. Then the covariant derivative $\nabla^{V}(A)$ associated to $A \in \mathscr{A}_{b}$ transforms as

$$
\nabla^{V}(\phi \cdot A)=\tilde{\phi}^{-1} \nabla^{V}(A) \tilde{\phi}
$$

Now

$$
\nabla^{E}(A)=\nabla^{S} \otimes I+I \otimes \nabla^{V}(A)
$$

and letting $\tilde{\phi}=I \otimes \tilde{\phi}$, we see that $\nabla^{E}(A)$ transforms as

$$
\nabla^{E}(\phi \cdot A)=\hat{\phi}^{-1} \nabla^{E}(A) \hat{\phi},
$$

since $\hat{\phi}$ acts only on the second factor. For the same reason, the representation $\phi \rightarrow \hat{\phi}$ is compatible with the decomposition $E=E^{+} \oplus E^{-}$for even $q$. For a choice of connection $A \in \mathscr{A}_{b}$, we have then a basic Dirac operator $\left(\not_{b}\right)_{A}$ depending on $A$, which for $A \rightarrow \phi \cdot A$ transforms as

$$
\left(\not D_{b}\right)_{\varphi \cdot A}=\hat{\phi}^{-1}\left(\not D_{b}\right)_{A} \hat{\phi}
$$

We refer to the property in (3.6) as the covariance of $\left(D_{b}\right)_{A}$. We also remark that throughout, the basic connection $A \in \mathscr{A}_{b}$ is varied with all metrics in question remaining fixed. 
The difference form $\delta_{A, \varphi}=\phi \cdot A-A$ is a form on $M$ with values in the adjoint bundle $\operatorname{Ad}(P)$ and from (3.3) we deduce that it is determined by

$$
\delta_{A, \varphi}(X)=\psi_{*}(\tilde{X}),
$$

where $\tilde{X}$ is the horizontal lift of a vectorfield $X$ relative to the connection $A$. As Clifford multiplication and $\hat{\phi}$ act on different parts of $E$, namely $S$ and $V,\left(D_{b}\right)_{A}$ and $\hat{\phi}$ commute up to a multiplication operator, i.e. the difference of the corresponding Dirac operators may be written as

$$
\left(D_{b}\right)_{\varphi \cdot A}-\left(D_{b}\right)_{A}=\hat{\phi}^{-1}\left[\left(D_{b}\right)_{A}, \hat{\phi}\right]=\Xi_{A, \phi},
$$

where $\Xi_{A, \phi}$ is a basic hermitian endomorphism of $E$, locally given by the formula

$$
\Xi_{A, \phi}(s \otimes v)=\sum_{\alpha}\left(E_{\alpha} \cdot s\right) \otimes \rho_{*} \psi_{*}\left(\tilde{E}_{\alpha}\right)(v) .
$$

We proceed by defining a principal bundle

$$
\left(\mathscr{A}_{b} \times P, \mathscr{G}(\tilde{\mathscr{F}}), \mathscr{A}_{b} \times_{\mathscr{G}(\tilde{\mathscr{F}})} P=\tilde{P}\right),
$$

where $\mathscr{G}(\tilde{\mathscr{F}})$ acts on $\mathscr{A}_{b} \times P$ by $(A, u) \rightarrow\left(\phi \cdot A, \phi^{-1}(u)\right)$. Now with our choice of $\mathscr{G}(\tilde{\mathscr{F}}), \widetilde{P}$ is itself a principal $G$-bundle

$$
G \rightarrow \tilde{P} \rightarrow \mathscr{A}_{b} / \mathscr{G}(\tilde{\mathscr{F}}) \times M
$$

With regards to defining a connection $\tilde{\omega}$ on $\tilde{P}$ and a formula for the curvature $\Omega_{\tilde{\omega}}$ pointwise at $([A], u)$, one may follow the details in [A-S4] practically verbatim; their space of connections and gauge group are replaced by our $\mathscr{A}_{b}$ and $\mathscr{G}(\widetilde{\mathscr{F}})$ respectively. Recalling the Atiyah sequence of vector bundles on $M$

$$
0 \rightarrow \operatorname{Ad}(P) \rightarrow T P / G \rightarrow T M \rightarrow 0,
$$

it is a straightforward matter to deduce that pointwise

$$
T\left(\mathscr{A}_{b} \times P\right) \cong \Omega_{b}^{1}(\mathscr{F}, \operatorname{Ad}(P)) \oplus T P .
$$

Using the fact that $\mathscr{G}(\tilde{\mathscr{F}})$ commutes with $G$ and applying $i_{\tilde{X}}$ to the components of type $(2,0),(1,1)$ and $(0,2)$ relative to $M \times \mathscr{A}_{b}$, one sees that $i_{\tilde{X}} \Omega_{\tilde{\omega}}=0$. This together with (3.13) shows that $\tilde{\omega}$ is itself basic (cf. [G1-K2]).

A particular motivation for introducing basic connections in gauge theory is provided by the following and is closely connected to the notion of dimension reduction of the pure Yang-Mills equations on $\mathbb{R}^{4}$ in the absolute case (see e.g. $[\mathrm{A}-\mathrm{H}],[\mathrm{J}-\mathrm{T}])$. We take $m=4$ and $q=3$ and select $\hat{A} \in \mathscr{A}$ such that $L_{\tilde{X}} \hat{A}=0$, i.e. $\hat{A}$ is invariant under the leaf flow. Let $X \in L(\mathscr{F})$, be a unit Killing vector field, $|X|=1$, so that the characteristic 1 -form $\chi_{\mathscr{F}}$ satisfies $\chi_{\mathscr{F}}(X)=1$. Setting $\varphi=i_{\tilde{X}} \hat{A}$, we have

$$
L_{\tilde{X}} \varphi=L_{\tilde{X}} i_{\tilde{X}} \hat{A}=-i_{\tilde{X}} L_{\tilde{X}} \hat{A}=0
$$

Now by definition we have $\varphi \in \operatorname{End}(V)$, and thus may regard $\varphi$ as a basic Higgs field. If we now write

$$
A=\hat{A}-\varphi \wedge \chi_{\mathscr{F}},
$$


we have $L_{\tilde{X}} A=-L_{\tilde{X}} \varphi \wedge \chi_{\mathscr{F}}-\varphi L_{\tilde{X}} \chi_{\mathscr{F}}=0$. From $i_{\tilde{X}} \chi_{\mathscr{F}}=1$, we also have

$$
i_{\tilde{X}} A=i_{\tilde{X}} \hat{A}-\varphi i_{\tilde{X}} \chi_{\mathscr{F}}=0 \text {, }
$$

hence showing that $A$ is basic, i.e. $A \in \mathscr{A}_{b}$. Thus associated to $\hat{A}$ is a basic vector potential $A$ together with a basic Higgs field $\varphi$ related by (3.14). As for the curvature, we may write

$$
F_{\hat{A}}=F_{A}+\Psi
$$

where $F_{A} \in \Omega_{b}^{2}(\mathscr{F}, \operatorname{Ad}(P))$ and $\Psi$ is an $\operatorname{Ad}(P)$-valued 2-form. In keeping with the analogous situation of $[\mathrm{A}-\mathrm{H}]$ and noting that in this case the mean curvature $\kappa=0$, one readily deduces from $F_{\hat{A}}=\mathrm{d} \hat{A}+\frac{1}{2}[\hat{A}, \hat{A}],(1.5)$ and (3.14) that

$$
\Psi=(d \varphi+[A, \varphi]) \wedge \chi_{\mathscr{F}}
$$

and

$$
i_{\tilde{X}} \Psi=-(d \varphi+[A, \varphi])=-D_{A} \varphi .
$$

Assuming self-duality $* F_{\hat{A}}=F_{\hat{A}}$, we may proceed further. Let $\bar{*}$ denote the transversal star operator, $\bar{*}: \Omega_{b}^{r}(\mathscr{F}) \rightarrow \Omega_{b}^{3-r}(\mathscr{F})$, where $*=\bar{*} \wedge \chi_{\mathscr{F}}$. Thus observing that $* F_{A}=\bar{*} F_{A} \wedge \chi_{\mathscr{F}}$, we deduce from (3.15), (3.17) and (1.5)

$$
* F_{\hat{A}}=\bar{*} F_{A} \wedge \chi_{\mathscr{F}}+\bar{*}\left(D_{A} \varphi\right) .
$$

Applying the self-duality condition, we now equate terms in (3.15) and (3.18) to derive a generalized Bogomolny equation (cf. $[\mathrm{A}-\mathrm{H}]$ ):

$$
F_{A}=\bar{*} D_{A} \varphi \text {. }
$$

The other equations characterizing the YMH system obtained by the above leaf-flow invariance of the self-dual YM equations on a 4-manifold, are easily deduced to be

$$
\begin{aligned}
D_{A} F_{A} & =0 \\
D_{A} \bar{*} F_{A} & =-\left[\varphi, D_{A} \varphi\right], \\
\mathrm{D}_{A} \bar{*} D_{A} \varphi & =0 .
\end{aligned}
$$

With regards to the sequence in (1.1), we see that if the second Stiefel-Whitney class $w_{2}(M)=0$, then automatically $w_{2}(Q)=0$, since $L(\mathscr{F})$ is trivial in this case and hence the above system may be equipped with a basic Dirac operator $D_{b}$. Since $q$ is odd, the analytic nature of the $D_{b}$ family is directly related to the notion of spectral flow (following [A-P-S]). This point will be taken up in Sect. 7.

As regards the heterotic string mentioned in the introduction, it is worthwhile to note that when $\left(M, \mathscr{F}, g_{M}\right)$ has compact leaves, the leaf space $M / \mathscr{F}$ is endowed with the structure of a $q$-dimensional orbifold [Mo]. This means that $M / \mathscr{F}$ is locally identifiable with $\bar{M} / \Gamma$, where $\bar{M}$ denotes a $q$-dimensional oriented manifold and $\Gamma$ is a finite group acting on $\bar{M}$. As in [A-B2], one may pass to the equivariant quotient

$$
\bar{M}_{\Gamma}=E \Gamma \times{ }_{\Gamma} \bar{M} .
$$

With the assumption $w_{2}(Q)=0$, we have a basic Dirac family which extends to $\bar{M}_{\Gamma}$ when the latter is viewed as a simplicial manifold. In the case corresponding 
to $q=10$ as described in $[F-V]$ (with $G=S O(32)$ or $E_{8} \times E_{8}$ ), the condition $w_{2}\left(\bar{M}_{\Gamma}\right)=0$ partially accounts for anomaly cancellation relative to the space $\operatorname{Map}\left(\Sigma, \bar{M}_{\Gamma}\right)$, where $\Sigma$ denotes a closed Riemann surface. Extending the $\not_{b}$ family to the equivariant quotient in our setting, may be similarly interpreted.

\section{The Basic Topological Families Index}

In Sects. 4 and 5 we assume that the codimension $q$ of $\mathscr{F}$ is even. As we mentioned previously, each $\left(\not_{b}^{+}\right)_{A}$ for a choice of $A \in \mathscr{A}_{b}$ is non-elliptic; however, we recall that its extension $\left(\not_{\mathrm{tr}}^{+}\right)_{A}$ is transversally elliptic. Moreover, the symbols of the operators $\left(\not_{b}^{+}\right)_{A}$ and $\left(\not_{\mathrm{tr}}^{+}\right)_{A}$ are equal and independent of $A$, namely given by Clifford multiplication $\xi$. for $\xi \in Q_{x}$. The operator $\left(D_{t r}^{+}\right)_{A}$, being transversally elliptic, thus possesses a well defined symbol class $\sigma_{\mathrm{tr}} \in K\left(Q^{*}\right)=K\left(B\left(Q^{*}\right), S\left(Q^{*}\right)\right)$ such that $\left.\sigma_{\mathrm{tr}}\right|_{s\left(Q^{*}\right)}$ is an isomorphism (here $Q^{*}$ denotes the complexified dual normal bundle of $\mathscr{F}$, i.e. $Q^{*}=\operatorname{Hom}(Q, \mathbb{C})$ ). We will use complex $K$-theory as completion over compact sets (as apparent in [A-S4]). The maps appearing below are the usual maps in $K$-theory, as defined e.g. in [A-S1] [A-S2], although our notation is slightly different.

We now establish some notation. We elect to denote $\mathscr{A}_{b} / \mathscr{G}(\tilde{\mathscr{F}})$ by $Y$ and $\mathscr{A}_{b} / \mathscr{G}(\tilde{\mathscr{F}}) \times M$ by $Z$. With regards to the representation $\rho$, we define an "auxiliary" vector bundle

$$
\mathscr{E}^{0}=\tilde{P} \times{ }_{\rho} \mathbb{C}^{r}
$$

over $Z$ with projections

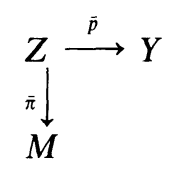

Now taking the complex spin bundle $S$ associated to $Q$ as a vector bundle over $M$, we define

$$
\mathscr{E}=\bar{\pi}^{*} S \otimes \mathscr{E}^{0}
$$

and extend the family $\left\{\left(D_{b}\right)_{A}\right\}$ to $\mathscr{E}$ as a family of operators $\left\{\left(\tilde{D}_{b}\right)_{A}\right\}$ on $\Gamma_{b}(\mathscr{E})$. For $q$ even, we similarly extend the family $\left\{\left(D_{b}^{ \pm}\right)_{A}\right\}$ to $\mathscr{E}^{ \pm}$as a family of operators $\left\{\left(\widetilde{D}_{b}^{ \pm}\right)_{A}\right\}: \Gamma_{b}\left(\mathscr{E}^{ \pm}\right) \rightarrow \Gamma_{b}\left(\mathscr{E}^{\mp}\right)$ over $Z$.

It will also be useful later to view the family $\left\{\left(\tilde{D}_{b}^{ \pm}\right)_{A}\right\}$ as an operator $\tilde{D}_{b}^{ \pm}$, acting along the fibers of the Hilbert bundle

$$
\mathscr{K}^{ \pm}=\mathscr{A}_{b} \times_{\mathscr{G}(\tilde{\mathscr{F}})} L^{2}\left(\Gamma_{b}\left(E^{ \pm}\right)\right) \rightarrow Y .
$$

Setting $\tilde{Q}^{*}=\bar{\pi}^{*} Q^{*}$, the element $\sigma_{\mathrm{tr}}$ may be lifted to an element $\tilde{\sigma}_{\mathrm{tr}}$ in $K\left(\tilde{Q}^{*}\right)$, and via the Thom isomorphism

$$
g_{!}: K(Z) \stackrel{\cong}{\longrightarrow} K\left(\tilde{Q}^{*}\right)
$$

defines an element $g_{!}^{-1}\left(\tilde{\sigma}_{\mathrm{tr}}\right)$ in $K(Z)$. The equality of the symbols of $\left(\not_{b}^{+}\right)_{A}$ and $\left(\not_{\mathrm{tr}}^{+}\right)_{A}$ motivates the following definition. 
Definition 4.1. The symbol class of the basic Dirac family $\left\{\left(\emptyset_{b}^{+}\right)_{A}\right\}$ is the element

$$
[\sigma]_{b}=g_{!}^{-1}\left(\tilde{\sigma}_{\mathrm{tr}}\right) \in K(Z) .
$$

Now that we have a well-defined symbol class, the definition of the basic topological families index is straightforward. We consider the homomorphism

$$
i_{!}: K(Z) \rightarrow K\left(Y \times \mathbb{C}^{N}\right)
$$

along with the periodicity isomorphism

$$
j_{!}: K(Y) \rightarrow K\left(Y \times \mathbb{C}^{N}\right) .
$$

Definition 4.2. The basic topological families index of $\left\{\left(D_{b}^{+}\right)_{A}\right\}$ is the element

$$
\operatorname{Ind}_{\text {top }}\left(\tilde{D}_{b}^{+}\right)=\left(j_{!}\right)^{-1}\left(i_{!}[\sigma]_{b}\right) \in K(Y) .
$$

This provides a homomorphism $K(Z) \rightarrow K(Y)$ and analogous to [A-S2], we deduce the formula

$$
\operatorname{ch}\left(\operatorname{Ind}_{\text {top }}\left(\tilde{D}_{b}^{+}\right)\right)= \pm \bar{p}_{*}\left(\operatorname{ch}\left([\sigma]_{b}\right) \wedge \operatorname{Td}\left(\tilde{Q}^{*}\right) \wedge \chi_{\mathscr{F}}\right)
$$

where $\operatorname{ch}()$ denotes the Chern character, $\operatorname{Td}\left(\tilde{Q}^{*}\right)$ the Todd class of $\tilde{Q}^{*}$ and $\bar{p}_{*}$ is integration along the fibers of $\bar{p}$ in (4.2). Noting that $\widetilde{Q}^{*}$ inherits a $\operatorname{Spin}(q)$-structure, we obtain from (4.3),

$$
\operatorname{ch}\left(g_{!}(\mathscr{E})\right)=g_{*}\left(\operatorname{ch}(\mathscr{E}) \wedge \operatorname{Td}\left(\tilde{Q}^{*}\right)\right)=g_{*}\left(\operatorname{ch}\left(\mathscr{E}^{0}\right) \wedge \hat{A}\left(\tilde{Q}^{*}\right)\right)
$$

where $\hat{A}$ denotes the (reduced) Atiyah-Hirzebruch class and

$$
g_{*}: H^{*}(Z, \mathbb{Q}) \stackrel{\cong}{\longrightarrow} H^{*}\left(\tilde{Q}^{*}, \mathbb{Q}\right)
$$

is the Thom isomorphism in rational cohomology. Together with (4.9), this yields the following formula for the Chern character of the topological families index

$$
\operatorname{ch}\left(\operatorname{Ind}_{\text {top }}\left(\tilde{D}_{b}^{+}\right)\right)= \pm \bar{p}_{*}\left(\operatorname{ch}\left(\mathscr{E}^{0}\right) \wedge \hat{A}\left(\tilde{Q}^{*}\right) \wedge \chi_{\mathscr{F}}\right)
$$

\section{The Basic Analytic Families Index}

Let $\mathscr{P}_{d}\left(Z ; \mathscr{E}^{+}, \mathscr{E}^{-}\right)$denote the space of pseudodifferential operators of order $d$ on $Z$ from $C^{\infty}\left(\mathscr{E}^{+}\right)$to $C^{\infty}\left(\mathscr{E}^{-}\right)$and let $\overline{\mathscr{P}}_{d}\left(Z ; \mathscr{E}^{+}, \mathscr{E}^{-}\right)$denote its Sobolev space completion with respect to $H_{s}\left(C^{\infty}\left(\mathscr{E}^{ \pm}\right)\right)$. Then in a standard way $\overline{\mathscr{P}}_{d}\left(Z ; \mathscr{E}^{+}, \mathscr{E}^{-}\right)$is a fiber bundle over $Z$ with fiber $\overline{\mathscr{P}}_{d}\left(M ; \mathscr{E}^{+}, \mathscr{E}^{-}\right)$. Regarding a section of $\overline{\mathscr{P}}_{d}\left(Z ; \mathscr{E}^{+}, \mathscr{E}^{-}\right)$as a family (in the sense of $[\mathrm{A}-\mathrm{S} 2]$ ), we see that the family $\left.\left\{\mathbb{D}_{\mathrm{tr}}^{+}\right)_{A}\right\}$, is given locally in the neighborhood of $A_{0} \in \mathscr{A}_{b}$ by a continuous map $\mathscr{A}_{b} \rightarrow \overline{\mathscr{P}}_{d}\left(M ; E^{+}, E^{-}\right)$. Passing to Sobolev spaces, this induces, locally, a continuous map

$$
\left(\not D_{b}^{+}\right)^{s}: \mathscr{A}_{b} \rightarrow \operatorname{Fred}\left(H_{s}\left(\Gamma_{b}\left(E^{+}\right)\right), H_{s-1}\left(\Gamma_{b}\left(E^{-}\right)\right)\right),
$$

where Fred denotes bounded Fredholm operators with the norm topology. Let $W=\operatorname{Ker}\left(\not_{b}^{+}\right)^{s}$. Then the map

$$
c_{s}(A): H_{s}\left(\Gamma_{b}\left(E^{+}\right)\right) \oplus W \rightarrow H_{s-1}\left(\Gamma_{b}\left(E^{-}\right)\right)
$$


defined by $c_{s}(A)(u \oplus v)=\left(\not_{b}^{+}\right)^{s}(u)+v$ is surjective for $A$ close to $A_{0}$. Now from the regularity property of the $\not_{b}^{+}$and Lemma (2.2), we see that $W \subset \Gamma_{b}\left(E^{-}\right)$and for $A$ close to $A_{0}$, the map $c(A): \Gamma_{b}\left(E^{+}\right) \oplus W \rightarrow \Gamma_{b}\left(E^{-}\right)$is surjective. The global argument in $\left[\mathrm{A}-\mathrm{S} 2\right.$, p. 126] now applies over $\mathscr{A}_{b}$, and we can define a surjective map

$$
\overline{\left(D_{b}^{+}\right)_{A}}: \Gamma_{b}\left(\mathscr{E}^{+}\right)_{A} \oplus \mathbb{C}^{k} \rightarrow \Gamma_{b}\left(\mathscr{E}^{-}\right)_{A}
$$

given by

$$
\left.\overline{\left(\not_{b}^{+}\right.}\right)_{A}\left(u ; v_{1}, \ldots, v_{k}\right)=\left(\not_{b}^{+}\right)_{A}(u)+\sum_{i=1}^{k} v_{i} s_{i}(A)
$$

for a finite number of sections $\left(s_{1}, \ldots, s_{k}\right)$ of $\Gamma_{b}\left(\mathscr{E}^{-}\right)$. The vector spaces $\operatorname{Ker}\left(\overline{D_{b}^{+}}\right)_{A}$ then comprise a vector bundle ker $\bar{D}_{b}^{+}$over $\mathscr{A}_{b}$ and we note that the element $\left[\operatorname{Ker} \bar{D}_{b}^{+}\right]-\left[\mathscr{A}_{b} \times \mathbb{C}^{k}\right]$ is a well defined element in $K\left(\mathscr{A}_{b}\right)$ and depends only on $\not_{b}^{+}$and not on the choice of sections $v_{i}$. As in [A-S4], the covariance property (3.6) implies $\operatorname{Ker}\left(\not_{b}^{+}\right)_{\phi \cdot A}=\phi^{-1}\left(\operatorname{Ker}\left(\not_{b}^{+}\right)_{A}\right)$ and permits us to define the above element as an element in $K(Y)$ :

Proposition 5.1. The element

$$
\left[\operatorname{Ker} \overline{D_{b}^{+}}\right]-\left[Y \times \mathbb{C}^{k}\right]
$$

is a well defined element of $K(Y)$.

As $Y$ is connected, we obtain by evaluation at $[A] \in Y$ :

Corollary 5.2. The index of the operators $\left(\not_{b}^{+}\right)_{A}$ is independent of $A \in \mathscr{A}_{b}$ :

$$
\text { Ind }\left(\left(D_{b}^{+}\right)_{A}\right)=c \in \mathbb{Z} \text {. }
$$

We refer to (5.5) as the basic analytic families index of the family $\left\{\left(D_{b}^{+}\right)_{A}\right\}$ and denote this by $\operatorname{Ind}_{a}\left(\tilde{D}_{b}^{+}\right)$. It remains to investigate the difference between (5.5) and (4.8), a measure of how the family $\left\{\left(\not_{b}^{+}\right)_{A}\right\}$ deviates from ellipticity for $q<m$.

Remark 5.3. As stated before, the transversal Bochner-Weitzenböck formulas (2.2) and (2.4) for $\left(\not_{b}\right)_{A}^{2}$, provide examples of operators with $\operatorname{ker}\left(\not_{b}\right)_{A}=0$ under suitable curvature conditions. In the present context $(E=S \otimes V)$, we find as in $[\mathrm{G}-\mathrm{L} 1,2]$, that $\mathscr{R}_{\nabla(A)}=\frac{1}{4} \sigma \otimes \mathrm{id}_{V}+\mathrm{id}_{S} \otimes \mathscr{R}_{A}$, where $\sigma$ denotes the transversal scalar curvature of the Levi-Civita connection in $Q$ and $\mathscr{R}_{A}$ depends only on the curvature in the coefficient bundle $V$ associated to the foliated bundle $P$. If we assume now that

$$
\mathscr{R}_{\nabla(A)}=\frac{1}{4} \sigma \otimes \mathrm{id}_{V}+\mathrm{id}_{S} \otimes \mathscr{R}_{A} \geqq 0,
$$

and strictly positive at a point $x \in M$, we find that $\operatorname{Ker}\left(\not_{b}^{ \pm}\right)_{A}=0$ and therefore by (3.6)

$$
\operatorname{Ind}\left(D_{b}^{+}\right)_{\phi \cdot A}=0
$$

along the orbit of $A \in \mathscr{A}_{b}$. It follows from (5.6) that Ind $\left(\not_{b}^{+}\right)_{A}=0$ for all $A \in \mathscr{A}_{b}$. This result is essentially due to Lichnerowicz [Li] in the absolute case.

\section{Maps from Y to Fredholm Operators}

The analytic families index parametrized by $\mathscr{A}_{b} / \mathscr{G}(\tilde{\mathscr{F}})=Y$ in the last section, essentially relates to the Hilbert bundles $\mathscr{K}^{ \pm}$over $Y$ in (4.4). Intuitively, the 
covariance condition in (3.6) implies that the family $\left\{\left(D_{b}^{ \pm}\right)_{\phi \cdot A}\right\}$, for $A$ fixed, gives an operator $\left(\not_{b}\right)_{\mathscr{G}(\tilde{\mathscr{F}}) \cdot A}$ on an orbit class mapping the fiber $\mathscr{K}_{\mathscr{G}(\tilde{\mathscr{F}}) \cdot A}$ to $\mathscr{K}_{\mathscr{G}(\tilde{\mathscr{F}}) \cdot \boldsymbol{A}}$.

We first recall a few well-known facts from functional analysis and homotopy theory (see $[\mathrm{F}-1][\mathrm{P}]$ ). Given a separable complex Hilbert space $H$, let $G L=(g \ell)^{*}$ be the group of units in the Banach algebra $g \ell=g \ell(H)$ of bounded linear operators on $H$ and $k \subset g \ell$ the ideal of compact operators. The inclusion $U=U(\infty) \subset G L_{\mathrm{cpt}} \equiv(I+k)^{*}$ of the weak limit $U(\infty)$ of the unitary groups $U(N)$, is a homotopy equivalence and $G L / G L_{\mathrm{cpt}} \cong(g \ell / k)_{0}^{*}$. The projection $g \ell \stackrel{\pi}{\rightarrow} g \ell / k$ to the Calkin algebra induces a homotopy equivalence between the space $\mathbf{F}=\operatorname{Fred}(H)=\pi^{-1}(g \ell / k)^{*}$ of Fredholm operators and the group of units $(g \ell / k)^{*}$. Using the fact that $G L$ is contractible (Kuiper's theorem), one obtains canonical homotopy equivalences $B U=G L / U \simeq B G L_{\mathrm{cpt}}=G L / G L_{\mathrm{cpt}} \simeq \mathbf{F}_{0}$, where $\mathbf{F}_{0}$ is the connected component of index 0 of $\mathbf{F}$.

For $q$ even, the construction of the analytic families index in Proposition 5.1 yields now a unique homotopy class of maps $\Psi_{(P, \tilde{F})}^{+}=\left[\Psi^{+}\right]$,

$$
\Psi^{+}: Y=\mathscr{A}_{b} / \mathscr{G}(\tilde{\mathscr{F}}) \rightarrow B U \times \mathbb{Z} \simeq G L / G L_{\mathrm{cpt}} \times \mathbb{Z} \simeq \mathbf{F},
$$

mapping $Y$ to the fixed component of index $c=\operatorname{Ind}\left(\left(D_{b}^{+}\right)_{A}\right)$ in $(5.6)(\mathrm{cf}$. $[\mathrm{A}-\mathrm{J}][\mathrm{A}-\mathrm{S} 4]$ [Si]).

A mapping in the class $\Psi_{(P, \tilde{\mathscr{F}})}^{+}$may be realized as follows. As $G L$ is contractible, the Hilbert bundle $\mathscr{K} \pm$ over $Y$ has a trivialization (unique up to homotopy), given by an equivariant mapping $s^{ \pm}: \mathscr{A}_{b} \rightarrow G L^{ \pm}=G L\left(L^{2}\left(\Gamma_{b}\left(E^{ \pm}\right)\right)\right)$satisfying

$$
S^{ \pm}(\phi \cdot A)=\left(\hat{\phi}^{ \pm}\right)^{-1} \circ S^{ \pm}(A) \text {. }
$$

The covariance condition (3.6) and formula (6.2) imply that the mapping

$$
\Psi^{+}(A)=S^{-}(A)^{-1} \circ\left(D_{b}^{+}\right)_{A} \circ S^{+}(A)
$$

is constant on $\mathscr{G}(\tilde{\mathscr{F}})$-orbits and thus defines

$$
\Psi^{+}: Y=\mathscr{A}_{b} / \mathscr{G}(\tilde{\mathscr{F}}) \rightarrow \operatorname{Fred}\left(H_{1}\left(\Gamma_{b}\left(E^{+}\right)\right), L^{2}\left(\Gamma_{b}\left(E^{-}\right)\right)\right)_{c} \simeq \mathbf{F}_{c}
$$

which evidently realizes the class $\Psi_{(\boldsymbol{P}, \tilde{F})}^{+}$.

We have a diagram of principal bundles with total spaces contractible:

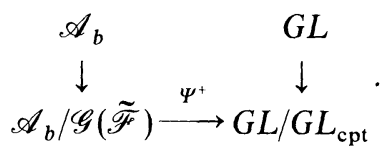

Passing to loop spaces $[\mathrm{P}-\mathrm{S}]$, we observe that $\mathscr{A}_{b} / \mathscr{G}(\tilde{\mathscr{F}}) \simeq B \mathscr{G}(\tilde{\mathscr{F}})$ and $G \simeq \Omega B G$ for any topological group. Thus we obtain a homotopy class $\Omega \Psi_{(P, \tilde{F})}^{+}=\left[\Omega \Psi^{+}\right]$of maps

$$
\Omega \Psi^{+}: \mathscr{G}(\tilde{\mathscr{F}}) \simeq \Omega B \mathscr{G}(\tilde{\mathscr{F}})=\Omega Y \rightarrow \Omega F_{c} \simeq \Omega B G L_{\mathrm{cpt}} \simeq G L_{\mathrm{cpt}},
$$

which, as in [Si], may be realized along a fixed orbit for which $\left(D_{b}^{+}\right)_{A}$ is invertible by the mapping

$$
\begin{aligned}
\Omega \Psi^{+}(\phi) & =\left(\not D_{b}^{+}\right)_{A}^{-1} \circ\left(\not D_{b}^{+}\right)_{\phi \cdot A} \\
& =\left(\not D_{b}^{+}\right)_{A}^{-1} \circ\left(\hat{\phi}^{-}\right)^{-1}\left[\left(\not_{b}^{+}\right)_{A}, \hat{\phi}\right]+I \\
& =\left(\not_{b}^{+}\right)_{A}^{-1} \circ \Xi_{A, \phi}^{+}+I,
\end{aligned}
$$


where the bundle map $\Xi_{A, \phi}^{+}$is determined by (3.8), (3.9) in an obvious sense. We summarize these constructions in the following theorems.

Theorem 6.1. There exist well-defined homotopy classes of maps $\Psi_{(P, \tilde{\mathscr{F}})}^{+}$from $B \mathscr{G}(\tilde{\mathscr{F}})$ to $B U \simeq \mathbf{F}_{c}$ and $\Omega \Psi_{(P, \tilde{F})}^{+}$from $\mathscr{G}(\tilde{\mathscr{F}})$ to $U \simeq G L_{\mathrm{cpt}}$, realized by (6.3), (6.7) such that the induced homomorphisms

$$
\Psi_{(P, \tilde{\mathscr{F}})}^{+*}: H^{*}(B U, \mathbb{Z}) \rightarrow H^{*}(B \mathscr{G}(\tilde{\mathscr{F}}), \mathbb{Z})
$$

and

$$
\Omega \Psi_{(P, \tilde{F})}^{+*}: H^{*}(U, \mathbb{Z}) \rightarrow H^{*}(\mathscr{G}(\tilde{\mathscr{F}}), \mathbb{Z})
$$

are related by transgression in the respective universal bundles. We have moreover,

$$
\Psi_{\left(\boldsymbol{P}, \tilde{F}_{)}\right)}^{+*}\left(\tilde{c}_{j}\right)=c_{j}\left(\operatorname{Ind}_{a}\left(\tilde{\mathscr{D}}_{b}^{+}\right)\right),
$$

where $\tilde{c}_{j}$ denotes the universal Chern class.

Theorem 6.2. In the category of foliated $G$-bundles $(P, \tilde{\mathscr{F}})$ over $(M, \mathscr{F})$ of fixed even codimension $q$, the homomorphisms (6.8) are compatible with pullbacks along $C^{\infty}$-mappings $f: M^{\prime} \rightarrow M$ transversal to $\mathscr{F}$; i.e. the pullback diagram

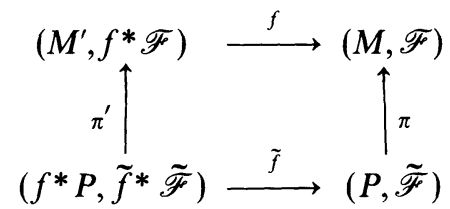

induces a canonical homomorphism $\bar{f}: \mathscr{G}(\tilde{\mathscr{F}}) \rightarrow \mathscr{G}\left(\tilde{f}^{*} \tilde{\mathscr{F}}\right)$ satisfying

$$
\Psi_{(P, \tilde{F})}^{+*}=B(\bar{f})^{*} \circ \Psi_{\left(\tilde{f}^{* P}, \tilde{f} \tilde{F}^{*} \tilde{\mathscr{F}}\right)}^{+*}
$$

Remarks 6.3.

(i) In Sect. 8 we will see how to interpret the characteristic class corresponding to $\Psi_{(P, \tilde{F})}^{+}\left(c_{1}\right) \in H^{2}(B \mathscr{G}(\tilde{\mathscr{F}}), \mathbb{Z})$, respectively its suspension in $H^{1}(\mathscr{G}(\tilde{\mathscr{F}}), \mathbb{Z})$, via the determinant map on $\mathscr{G}(\tilde{\mathscr{F}})$, i.e. as an obstruction to defining a gauge invariant determinant for the family $\left\{\left(D_{b}^{+}\right)_{A}\right\}$. In analogy with the absolute case, one might therefore call these classes $\mathscr{F}$-relative chiral anomalies.

(ii) We remark that for the absolute case $(q=m)$ the transversality condition implies that $f$ must be a submersion. In this case $L\left(f^{*} \mathscr{F}\right)=T(f)$, the tangent bundle along the fibers of $f$, and $f^{*} \mathscr{F}$ is given by the (connected components) of the fibers of $f$.

For odd codimension $q$, the spin representation $\Delta_{s}$ is irreducible, but the family $\left\{\left(D_{b}\right)_{A}\right\}$ may be used to construct a map from $Y$ to bounded self-adjoint Fredholm operators. We trivialize the Hilbert bundle

$$
\mathscr{K}=\mathscr{A}_{b} \times_{\mathscr{G}(\tilde{\mathscr{F}})} L^{2}\left(\Gamma_{b}(E)\right) \rightarrow Y
$$

by an equivariant map s: $\mathscr{A}_{b} \rightarrow G L=G L\left(L^{2}\left(\Gamma_{b}(E)\right)\right)$ and then set

$$
\hat{\Psi}(A)=s(A)^{-1} \circ\left(D_{b}\right)_{A} \circ S(A) .
$$

This defines as before a canonical map

$$
\hat{\boldsymbol{\Psi}}: Y \simeq B \mathscr{G}(\tilde{\mathscr{F}}) \rightarrow \hat{\mathbf{F}}_{*} \subset \operatorname{Fred}_{\mathrm{sa}}\left(H_{1}\left(\Gamma_{b}(E)\right), L^{2}\left(\Gamma_{b}(E)\right)\right),
$$


which, at least under some mild restrictions on $\mathscr{F}$, takes values in the non-trivial component $\hat{\mathbf{F}}_{*} \simeq \Omega \mathbf{F} \simeq \Omega B U \simeq U$ of self-adjoint Fredholm operators [A-S3]; we denote its homotopy class by $\hat{\Psi}_{(\boldsymbol{P}, \tilde{\mathscr{F}})}$. This is in accordance with the index class of a self-adjoint family being an element of $K^{-1}(Y)$ [A-P-S, III], [A-S3].

Passing again to loop spaces, one obtains a homotopy class $\Omega \hat{\Psi}_{(P, \tilde{F})}$ of maps

$$
\Omega \hat{\Psi}: \mathscr{G}(\tilde{\mathscr{F}}) \simeq \Omega B \mathscr{G}(\tilde{\mathscr{F}}) \simeq \Omega Y \rightarrow \Omega \hat{\mathbf{F}}_{*} \simeq \Omega^{2} \mathbf{F} \simeq \mathbf{F} \simeq B U \times \mathbb{Z}
$$

by Bott periodicity. Thus in the odd case, the situation is exactly reversed and one obtains cohomology homomorphisms (cf. [Si])

$$
\Psi_{(P, \tilde{\mathscr{F}})}^{*}: H^{*}(U, \mathbb{Z}) \rightarrow H^{*}(B \mathscr{G}(\tilde{\mathscr{F}}), \mathbb{Z})
$$

and

$$
\Omega \hat{\Psi}_{(P, \tilde{\mathscr{F}})}^{*}: H^{*}(B U, \mathbb{Z}) \rightarrow H^{*}(\mathscr{G}(\tilde{\mathscr{F}}), \mathbb{Z}) .
$$

In the next section, we give an explicit realization of a map in the class $\Omega \hat{\Psi}_{(P, \tilde{F})}$ by suspending a gauge transformation to obtain a periodic family of self-adjoint basic Dirac operators. The homomorphism

$$
\hat{\Psi}_{*}: \pi_{0}(\mathscr{G}(\tilde{\mathscr{F}})) \cong \pi_{1}(Y) \rightarrow \pi_{1}\left(\hat{\mathbf{F}}_{*}\right) \cong \pi_{0}(\mathbf{F}) \cong \mathbb{Z}
$$

may then be interpreted as the spectral flow of the periodic family $[\mathrm{A}-\mathrm{P}-\mathrm{S}, \mathrm{III}]$.

Remark 6.4. The homotopy class $\hat{\Psi}_{(P, \tilde{\mathscr{F}})}^{2}$ corresponding to the family $\left(\not_{b}\right)_{A}^{2}$ may be realized by the map

$$
\hat{\Psi}^{2}(A)=s(A)^{-1} \circ \Psi_{\zeta}(A) \circ S(\mathrm{~A}),
$$

where $\Psi_{\zeta}(A)=I+\zeta R\left(\left(D_{b}\right)_{A}^{2}, \zeta\right), \zeta=-1$, is the bounded self-adjoint Fredholm operator defined by the resolvent in remark 2.4 (ii). As these operators are non-negative, $\hat{\boldsymbol{\Psi}}^{2}$ takes values in the contractible component $\hat{\mathbf{F}}^{+}$of essentially positive operators.

\section{Spectral Flow of the Basic Dirac Family}

In this section we generalize part of the results of Vafa and Witten in [V-W] concerning uniform bounds on the eigenvalues $\mu_{j}$ of Dirac operators coupled to vector potentials, to the foliation context. The description by Atiyah in [A2, Theorem $1^{*}$ ] and the essential geometric framework suit our basic Dirac operators quite appropriately.

Let $E=S \otimes V$ be a foliated twisted Spin bundle as in (3.1). We recall that $\left(\not_{b}\right)_{A}$ has a discrete spectrum and assume that the eigenvalues $\mu_{j}(A)$ are ordered by increasing values, counting multiplicities, i.e. $\operatorname{Spec}\left(\left(D_{b}\right)_{A}\right)=\left\{\mu_{j} \in \mathbb{R}, \mu_{j} \leqq \mu_{j+1}\right.$, $j \in \mathbb{Z}\}$.

Given a gauge transformation $\phi \in \mathscr{G}(\tilde{\mathscr{F}})$, we suspend the automorphism $\phi$ by considering the mapping torus

$$
\bar{E}=\mathbb{R} \times{ }_{\phi} E \rightarrow S^{1} \times M,
$$

i.e. by identifying $(t+n, s) \sim\left(t, \hat{\phi}^{n}(s)\right), s \in E, t \in \mathbb{R}, n \in \mathbb{Z}$. Sections of $\bar{E}$ are given by 
those sections of $\mathbb{R} \times E \rightarrow \mathbb{R} \times M$ which satisfy the identities

$$
s(t+n, x)=\hat{\phi}^{-n} s(t, x) \text {. }
$$

It is clear that $\vec{E} \rightarrow S^{1} \times M$ is a foliated twisted Spin bundle with respect to the foliation $\overline{\mathscr{F}}$ on $S^{1} \times M$ of codimension $(q+1)$, given by $L(\overline{\mathscr{F}})=0 \times L(\mathscr{F})$. We define a 1 -parameter family $\left\{\mathrm{D}_{t}\right\}_{\mathrm{t} \in \mathbb{R}}$ of basic Dirac operators for a given $A \in \mathscr{A}_{b}$ by

$$
\mathrm{D}_{t}=\left(\not_{b}\right)_{A_{t}},
$$

where

$$
A_{t}=(1-(t-n)) \phi^{n} \cdot A+(t-n) \phi^{n+1} \cdot A=\phi^{n} \cdot A_{t-n}
$$

for $t \in[n, n+1], n \in \mathbb{Z}$. The covariance condition (3.6) is now expressed by the following periodicity condition for the family $\left\{D_{t}\right\}_{\mathrm{t} \in \mathbb{R}}$ :

$$
\mathrm{D}_{t+1}=\hat{\phi}^{-1} \circ \mathrm{D}_{t} \circ \hat{\phi} \text { for } t \in \mathbb{R} \text {. }
$$

This defines therefore an operator $\overline{\mathrm{D}}$, acting along the fibers of the Hilbert bundle

$$
\mathscr{K}_{\phi}=\mathbb{R} \times{ }_{\phi} L^{2}\left(\Gamma_{b}(E)\right) \rightarrow S^{1} .
$$

In fact, $\mathscr{K}_{\phi}, \bar{D}$ and $\bar{E}$ are exactly the pull back of the "universal" quantities $\mathscr{K}$, $\widetilde{D}_{b}$ and $\mathscr{E}$ in (4.3), (6.13) via the mapping $\bar{A}: S^{1} \times M \rightarrow Y \times M$ defined by $\bar{A}(t, x)=\left(\left[A_{t}\right], x\right)$ (notice that $\left[A_{t}\right], t \in[0,1]$ defines a loop in $\left.Y=\mathscr{A}_{b} / \mathscr{G}(\tilde{\mathscr{F}})\right)$.

Using (3.8), (3.9), we may rewrite the periodic family $\left\{D_{t}\right\}_{t \in \mathbb{R}}$ as

$$
\mathrm{D}_{t}=\left(\not D_{b}\right)_{A}+t \Xi_{A, \phi}, \text { for } t \in[0,1] \text {, }
$$

or via $(7.5)$,

$$
\mathrm{D}_{t}=\hat{\phi}^{-n_{\circ}}\left(\left(D_{b}\right)_{A}+(t-n) \Xi_{A, \phi}\right) \circ \hat{\phi}^{n}
$$

for $t \in[n, n+1], n \in \mathbb{Z}$.

Further, as the representation $\phi \rightarrow \hat{\phi}$ of $\mathscr{G}(\tilde{\mathscr{F}})$ in $G L=G L\left(L^{2}\left(\Gamma_{b}(E)\right)\right)$ is unitary [A-B1], [M-R], it follows from the periodicity condition (7.5) that $\mathrm{D}_{t+1}$ and $\mathrm{D}_{t}$, are unitarily equivalent for $t \in \mathbb{R}$ and so

$$
\operatorname{Spec}\left(D_{t+1}\right)=\operatorname{Spec}\left(D_{t}\right), \text { for } t \in \mathbb{R} \text {. }
$$

This construction, assigning to a gauge transformation $\phi$ a loop in $Y$ and a periodic family in $\hat{\mathbf{F}}_{*}$, realizes a map in the homotopy class $\Omega \hat{\Psi}_{(P, \tilde{F})}$ in $(6.16)$. The induced homomorphism

$$
\pi_{1}\left(S^{1}\right) \cong \mathbb{Z} \rightarrow \pi_{1}(Y) \rightarrow \pi_{1}\left(\hat{\mathbf{F}}_{*}\right) \cong \pi_{0}(\mathbf{F}) \cong \mathbb{Z}
$$

determines an integer, which we define as the basic spectral flow $\operatorname{sf}_{b}(\bar{D})$ of the periodic family $\bar{D}=\left\{\mathrm{D}_{t}\right\}_{\mathrm{t} \in \mathbb{R}}$. This notion of spectral flow, as introduced in [A-P-S, III], can be developed in the present context just as in the absolute case. Intuitively, it may be thought of as a monodromy invariant in the Hilbert bundle (7.6), as the solutions of the eigenvalue equations move around $S^{1}$, thereby inducing a shift on $\operatorname{Spec}\left(\left(\not_{b}\right)_{A}\right)$. The operator $\mathscr{D}_{b}=\mathrm{D}_{t}+\partial / \partial t$, with adjoint $\mathscr{D}_{b}^{*}=\mathrm{D}_{t}-\partial / \partial t$, is well defined on the (basic) sections of $E$ by (7.2) and (7.5). The fundamental relationship of the spectral flow of $\bar{D}$ with the index of $\mathscr{D}_{b}$ remains valid. If the codimension of $\mathscr{F}$ is odd, then one has the following formula for the basic spectral 
flow of the family $\bar{D}$ (cf. $[\mathrm{A}-\mathrm{P}-\mathrm{S}, \mathrm{III}]$ ):

$$
\operatorname{sf}_{b}\left(\bar{D}_{b}\right)=\operatorname{Ind}_{a}\left(\mathscr{D}_{b}\right) .
$$

We use this construction, as in [A2], to prove the following theorem (cf. $[\mathrm{V}-\mathrm{W}])$.

Theorem 7.1. Assume that $(M, \mathscr{F})$ is of odd codimension $q=2 l+1$ and admits a foliated $C^{\infty}$-mapping (constant on the leaves of $\left.\mathscr{F}\right) f: M \rightarrow S^{q}$ inducing an oriented isomorphism

$$
f^{*}: H^{q}\left(S^{q}, \mathbb{R}\right) \stackrel{\cong}{\longrightarrow} H^{q}\left(\Omega_{b}(\mathscr{F})\right),
$$

i.e. $f^{*}(\mu)=c v, c>0$, where $\mu$ denotes the orientation class of $S^{q}$ and $v$ the cohomology class defined by the transversal Riemannian volume form in $H^{q}\left(\Omega_{b}(\mathscr{F})\right)$. Then there exists a constant $C_{1}>0$, independent of $A$ and $V$, such that any interval $\left[a-C_{1}, a+C_{1}\right], a \in \mathbb{R}$, contains at least one eigenvalue of $\left(\mathbb{D}_{b}\right)_{A}$ for all $A \in \mathscr{A}_{b}$, i.e.

$$
\operatorname{Spec}\left(\left(D_{b}\right)_{A}\right) \cap\left[a-C_{1}, a+C_{1}\right] \neq \emptyset .
$$

Proof. It is clearly sufficient to prove the theorem for $V \otimes \mathbb{C}^{N} \cong N V, N>1$, and connections of the form $N A$ on $N V$, as the eigenvalues of $\left(\not_{b}\right)_{N A}$ are evidently those of $\left(D_{b}\right)_{A}$ with $N$ times their multiplicity.

The following argument is based on the outline in [A2], adapted to our present context. Choose $\psi: S^{q} \rightarrow U(N)$, representing a generator of $\pi_{q}(U)(N$ in the stable range). Then $\varphi=\psi \circ f: M \rightarrow U(N)$ defines a gauge transformation in $M \times \mathbb{C}^{N}$ and hence in $V \otimes \mathbb{C}^{N} \cong N V$. We denote by $\hat{\varphi}=I \otimes \varphi$ the resulting gauge transformation in $N E=E \otimes \mathbb{C}^{N}=S \otimes\left(V \otimes \mathbb{C}^{N}\right)$ (observing that we may as well take $G=U(r)$ and for $\rho$ the representation of $U(r)$ by diagonal blocks in $U(r N))$. We have that $\varphi \in \mathscr{G}(\tilde{\mathscr{F}})$ by Proposition 3.1 (i) and (7.12). Setting $A_{0}=A \otimes I=N A$, we obtain therefore by the previous construction (7.3), (7.4) a 1-parameter family $\bar{D}=\left\{\mathrm{D}_{t}\right\}_{1 \in \mathbb{R}}$ of basic Dirac operators satisfying the periodicity condition (7.5). From (3.8), (3.9) and (7.7), we find now that

$$
\left[\left(D_{b}\right)_{A_{0}}, \hat{\varphi}\right]=\hat{\varphi} \Xi_{\varphi},
$$

where the hermitian multiplication operator $\Xi_{\varphi}: T M \rightarrow \mathfrak{u}(N)$ is given by

and

$$
\Xi_{\varphi}(s \otimes v)=\sum_{\alpha}\left(E_{\alpha} \cdot s\right) \otimes \varphi^{-1}\left(E_{\alpha} \varphi\right)(v),
$$

$$
\mathrm{D}_{t}=\left(\not_{b}\right)_{A_{0}}+t \Xi_{\varphi}, \text { for } t \in[0,1] \text {. }
$$

In contrast to the general situation in (7.7), the endomorphism $\Xi_{\varphi}$ is now independent of $A$ and $V$. This important fact is the source for the uniformity in the estimate (7.13).

If $\operatorname{ker}\left(\left(D_{b}\right)_{A}-a I\right) \neq 0$, then the assertion of the theorem is trivially true and thus we may assume that $a \notin \operatorname{Spec}\left(\left(D_{b}\right)_{A}\right)$.

Using the explicit construction of the gauge transformation $\varphi$ and the family $\bar{D}$, one checks directly that it has spectral flow $s f_{b}(\bar{D}) \neq 0$. Thus an eigenvalue must cross the gap at $a$ as $t: 0 \rightarrow 1$ and there exists $t_{0} \in(0,1)$ such that $a \in \operatorname{Spec}\left(D_{t_{0}}\right)$, i.e. $\operatorname{ker}\left(\mathrm{D}_{t_{0}}-a I\right) \neq 0$. We claim that the desired constant $C_{1}>0$ is now given by 
$C_{1}=\left\|\Xi_{\varphi}\right\|$. In fact, using the variational characterization of the eigenvalue $\mu\left(A_{0}\right)$ of $\mathrm{D}_{0}=\left(D_{b}\right)_{A_{0}}$ closest to $a$ by

$$
\left(\mu\left(A_{0}\right)-a\right)^{2}=\inf _{\|s\|=1}\left\langle\left(\mathrm{D}_{0}-a I\right)^{2} s, s\right\rangle=\inf _{\|s\|=1}\left\|\left(\mathrm{D}_{0}-a I\right) s\right\|^{2},
$$

we find for $s \in \operatorname{ker}\left(\mathrm{D}_{t_{0}}-a I\right),\|s\|=1$ by (7.16):

$$
\left|\mu\left(A_{0}\right)-a\right| \leqq\left\|\left(\mathrm{D}_{0}-a I\right) s\right\|=\left\|\left(\mathrm{D}_{0}-\mathrm{D}_{t_{0}}\right) s\right\| \leqq\left\|\Xi_{\varphi}\right\| .
$$

The result follows.

Theorem 7.2. Assume that $(M, \mathscr{F})$ satisfies condition (7.12). Then there exists a constant $C_{0}>0$, independent of $A$ and $V$, such that the absolutely smallest eigenvalue $\mu_{1}(A)$ of $\left(\not_{b}\right)_{A}$ satisfies

$$
\left|\mu_{1}(A)\right| \leqq C_{0} .
$$

Proof. For $q$ even and $\operatorname{Ind}\left(\not_{b}^{+}\right)_{A} \neq 0$, the theorem is immediate as noted before. In fact, we must then have $\operatorname{ker}\left(\left(D_{b}\right)_{A}\right) \neq 0$ and $0 \in \operatorname{Spec}\left(\left(D_{b}\right)_{A}\right)$.

For $q$ odd, the assertion of the theorem is a direct consequence of Theorem 7.1. For $q$ even, the foliation $\overline{\mathscr{F}}$ on $S^{1} \times M$, given by $L(\overline{\mathscr{F}})=0 \times L(\mathscr{F})$ satisfies condition (7.12) and the assertion of the theorem follows from Theorem 7.1 applied to the basic Dirac operators $\left(D_{b}\right)_{A}-i \partial / \partial t$ on $S^{1} \times E \rightarrow S^{1} \times M$, whose eigenvalues are $\pm\left(\mu_{j}^{2}+m^{2}\right)^{1 / 2}, m \in \mathbb{Z}$.

\section{Remarks 7.3.}

(i) We observe that condition (7.12) above implies that $H^{q}\left(\Omega_{b}(\mathscr{F})\right) \cong \mathbb{R}$ and hence that $\left(M, \mathscr{F}, g_{M}\right)$ is minimalizable or taut, i.e. $\kappa \sim 0$ (cf. $\left.[\mathrm{K}-\mathrm{T} 2,3,4]\right)$.

(ii) A condition like (7.12) in Theorem 7.1 is clearly required. It excludes cases like Lie $G$-foliations with dense leaves [Mo], where $\Omega_{b}(\mathscr{F}) \cong \Lambda \mathrm{g}^{*}$ is finite dimensional and therefore Theorem 7.1 cannot hold. It is not clear whether such a condition is required for the statement in Theorem 7.2 to be valid.

Let $g_{Q}$ denote the holonomy invariant metric on $Q$, recalling that $g_{M}$ is bundle-like [Re2]. Rescaling $g_{Q}$ as $g_{Q} \rightarrow t^{2} g_{Q}$, the transversal volume $\|v\|$ rescales as $\|v\| \rightarrow t^{q}\|v\|$ with the eigenvalues of $D_{b}$ in turn rescaling as

$$
\left|\mu_{j}\right| \rightarrow t^{-1}\left|\mu_{j}\right|=\left|\mu_{j}\right|\|v\|^{-q} \text {. }
$$

Then from the above inequality in Theorem 7.2 we deduce that

$$
\left|\mu_{1}\right| \leqq C_{0}\|v\|^{-1 / q} \text {. }
$$

As an application, let us take $q=4$ analogous to [V-W ]. Recalling the representation $\rho: G \rightarrow S U(r)$. Consider (configuration) variables $x_{1}, \ldots, x_{k}, k$ even, $k \leqq r$. Let $\psi_{1}, \ldots, \psi_{r}$ be local sections of $E=S \otimes V$, i.e. transversal fermions with coefficients in $V$ transforming with respect to $\rho$. We now define the integral, for $A \in \mathscr{A}_{b}$,

$$
\begin{aligned}
I_{A}(k) & =i^{(k+2)}\|v\|^{-1} \int d^{4} x_{1} \ldots d^{4} x_{k}\left\langle\bar{\psi}_{1} \psi_{2}\left(x_{1}\right) \ldots \bar{\psi}_{k} \psi_{1}\left(x_{k}\right)\right\rangle^{A} \\
& =i^{k}\|v\|^{-1} \int d^{4} x_{1} \ldots d^{4} x_{k} \operatorname{Tr} E^{A}\left(x_{1}, x_{2}\right) \ldots E^{A}\left(x_{k}, x_{1}\right) \\
& =\|v\|^{-1} \operatorname{Tr}\left(\left(i D_{b}\right)^{-k}\right)
\end{aligned}
$$


where $E^{A}\left(x_{i}, x_{j}\right)$ are propagators for $\not_{b}$. Consider the eigenvalue problem

$$
\left(i D_{b}\right) \psi=\mu \psi,
$$

where we use the ordering $\left|\mu_{1}\right| \leqq\left|\mu_{2}\right| \leqq \ldots$. Then (7.21) is expressible as

$$
I_{A}(k)=\|v\|^{-1} \sum_{i=1}^{\infty} \mu_{i}^{-k}
$$

whence we obtain

$$
I_{A}(k) \geqq\|v\|^{-1}\left(\left|\mu_{1}(A)\right|\right)^{-1 / 4} .
$$

Now applying (7.20) with $q=4$, we have

$$
I_{A}(k) \geqq C_{0}^{-k}\|v\|^{(k-4) / 4}
$$

which for $k>4$, diverges as $\|v\| \rightarrow \infty$. When the coefficient bundle $V$ is trivial (corresponding to the massless case), we obtain on averaging

$$
I(k) \geqq C_{0} I_{0}(k) .
$$

In [V-W ], the analogue of (7.25) has the QCD interpretation of absence of mass gap implying that under appropriate conditions $I(k)$ has the same infrared divergence as $I_{0}(k)$.

\section{Defining the Determinant and the Zeta Function Regularity}

Let

$$
N_{b}(t)=\#\left\{\lambda \leqq t: \lambda \in \operatorname{spec}\left(\not_{b}^{2}\right)\right\}
$$

be the counting function of $\operatorname{spec}\left(D_{b}^{2}\right)$ and $N(t)$ the corresponding counting function for spec $(\Delta)$. By using (2.5), the a priori elliptic estimates and the Sobolev lemma (cf. e.g. [Gi]) we have that $N(t)$ and $N_{b}(t)$ are polynomially bounded and that in fact $[\mathrm{B}-\mathrm{H} 1,2]$

$$
N_{b}(t) \leqq N(t) \sim c t^{m / 2}, \text { for } t \uparrow \infty .
$$

More precisely, there exists $q^{\prime}, 0 \leqq q^{\prime} \leqq q$, determined by the structure of the foliation, such that $[\mathrm{B}-\mathrm{K} 2]$ :

$$
N_{b}(t) \sim c_{b} t^{q^{\prime} / 2}, \text { for } t \uparrow \infty .
$$

Let $h_{b, t}=\operatorname{Tr}\left(e^{-t \Phi_{b}^{2}}\right)<\infty$, for $t>0$, where

$$
\operatorname{Tr}\left(e^{-t \phi_{b}^{2}}\right)=\sum_{j} e^{-\lambda_{j} t}
$$

Observe that $h_{b, t}$ is finite by (8.2). It follows from (8.3) by an abelian argument (cf. $[\mathrm{B}-\mathrm{H} 1,2]$ ), that

i.e.

$$
h_{b}(t) \sim a_{b} t^{-q^{\prime} / 2}, \text { for } t \downarrow 0,
$$

$$
t^{q^{\prime} / 2} h_{b}(t) \rightarrow a_{b}, \quad \text { for } \quad t \downarrow 0 .
$$


In the following, we assume that $\operatorname{ker}\left(\not_{b}^{2}\right)=0$ (e.g. by adding a positive multiple of the identity). We define the basic $\zeta$-function by

$$
\zeta_{b}(s)=\operatorname{Tr}\left(\left(D_{b}^{2}\right)^{-s}\right)=\sum_{j} \lambda_{j}^{-s}
$$

It follows from (8.2), respectively (8.3), that (8.6) is finite and that $\zeta_{b}(s)$ is holomorphic for $\operatorname{Re}(s)>q^{\prime} / 2$. We recall e.g. from $[\mathrm{A}-\mathrm{B}-\mathrm{P}]$, [Gi] that $h_{b, t}$ and $\zeta_{b}(s)$ are related by the Mellin transform

$$
\zeta_{b}(s) \Gamma(s)=\int_{0}^{\infty} t^{s} h_{b, t} d \log t
$$

In general one would have to take into account the possibility of multiple poles arising from logarithmic terms in an asymptotic expansion of $\zeta_{b}(s)$ for $t \downarrow 0$, as in the case of orbit spaces for compact group actions [B-H2]. However, in certain instances these terms do not arise (see [B-Sc]). Whether or not they actually do arise in other cases, remains an open question. Assuming that $h_{b, t}$ has an asymptotic expansion without logarithmic terms for $t \downarrow 0$ it follows from (8.3), (8.5) that the expansion must be of the form

$$
h_{b, t} \sim \sum_{k \geqq-q}^{\infty} a_{b, k} t^{k / 2}, \quad \text { as } t \downarrow \downarrow 0,
$$

i.e. the singular exponent is $-q^{\prime} / 2$. From (8.6), (8.7) one obtains then by a standard estimate for $\operatorname{Re}(s)>q^{\prime} / 2$,

$$
\begin{aligned}
\zeta_{b}(s) \Gamma(s)=\int_{0}^{\infty} t^{s} h_{b, t} d \log t & =\lim _{\varepsilon \downarrow 0} \int_{\varepsilon}^{\infty} t^{s} h_{b, t} d \log t \\
& =\sum_{k \geqq-q^{\prime}}^{N} \int_{0}^{\varepsilon} a_{k} t^{k / 2} t^{s} d \log t+\int_{\varepsilon}^{\infty} t^{s} h_{b, t} d \log t+o\left(\varepsilon^{s+\alpha+N / 2}\right) \\
& =\sum_{k \geqq-q^{\prime}}^{N} a_{k} \frac{\varepsilon^{s+k / 2}}{s+k / 2}+\int_{\varepsilon}^{\infty} t^{s} h_{b, t} d \log t+o\left(\varepsilon^{s+\alpha+N / 2}\right), \quad \alpha>0 .
\end{aligned}
$$

Hence $\zeta_{b}(s) \Gamma(s)$ is meromorphic with simple poles at $s_{k}=-k / 2, k \geqq-q^{\prime}$ and residues $a_{k}$. In particular, $\zeta_{b}(s)$ is then holomorphic at $s_{2 n}=-n$, with $a_{2 n}=\zeta_{b}(-n)$, $n \geqq 0[\mathrm{Se}]$.

For $q$ odd, we may now consider, formally

$$
\zeta_{b}^{\prime}(0)=\left.\frac{d}{d s}\right|_{s=0} \zeta_{b}(s)=\left.\frac{d}{d s}\right|_{s=0} \sum_{j} \lambda_{j}^{-s}=-\sum_{j} \log \lambda_{j}
$$

which may be computed, assuming (8.8), as in [Si] by

$$
\zeta_{b}^{\prime}(0)=\left.\left[\zeta_{b}(s) \Gamma(s)-\zeta_{b}(0) / s\right]\right|_{s=0}-\zeta_{b}(0) g(0),
$$

where $g(s)=\Gamma(s)-1 / s$. Thus

$$
\operatorname{det}\left(\left(D_{b}\right)_{A}^{2}\right)=\exp \left(-\zeta_{b, A}^{\prime}(0)\right)
$$

is a gauge-invariant definition of a determinant for $\left(D_{b}\right)_{A}^{2}, A \in \mathscr{A}_{b}$, since $\zeta_{b, A}(s)=\zeta_{b, \phi \cdot A}(s)$ by covariance (3.6). 
In the case where $q$ is even, we deduce from Theorem 2.3(iv) and (8.8) that

$$
\text { Ind }\left(\left(\not_{b}^{+}\right)_{A}\right)=a_{0}^{+}-a_{0}^{-}=\zeta_{b, A}^{+}(0)-\zeta_{b, A}^{-}(0),
$$

where $\zeta_{b, A}^{ \pm}(s)$ is the $\zeta$-function of $\left(D_{b}^{\mp}\right)_{A}{ }^{\circ}\left(D_{b}^{ \pm}\right)_{A}[\mathrm{~A}-\mathrm{B}-\mathrm{P}]$, [Se]. In general, it is not possible to define a gauge-invariant determinant for the basic Dirac operators $\left(D_{b}^{+}\right)_{A}$, except when $G$ is abelian. To proceed, we choose an operator $\left(D_{b}\right)_{A_{0}}$, satisfying $\operatorname{ker}\left(\not_{b}^{-}\right)_{A_{0}}=0$, and then identity $\Gamma_{b}\left(E^{-}\right)$as a subspace of $\Gamma_{b}\left(E^{+}\right)$of finite codimension via the fixed operator $\left(\not_{b}^{-}\right)_{A_{0}}$.

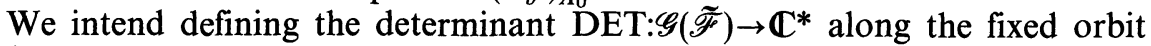
$\left\{\left(D_{b}^{+}\right)_{\phi \cdot A_{0}}\right\}_{\phi \in \mathscr{G}(\tilde{\mathscr{F}})}$ as an obstruction to a gauge-invariant determinant for $\left(D_{b}^{+}\right)_{\phi} \cdot A_{0} \cdot$ Setting $A_{0}=A$, we define the operator

$$
T_{\phi}=\left(\not D_{b}^{-}\right)_{A} \circ\left(D_{b}^{+}\right)_{\phi \cdot A}=\left(\not D_{b}^{-}\right)_{A}\left(\hat{\phi}^{-}\right)^{-1}\left(\not_{b}^{+}\right)_{A} \hat{\phi}^{+}: \Gamma_{b}\left(E^{+}\right) \rightarrow \Gamma_{b}\left(E^{+}\right)
$$

as a function on $\mathscr{G}(\tilde{\mathscr{F}})$. As in [A-S4], [Si], we require log DET to be a local function of $\phi \cdot A$. Again, there is the question of definability of $\log \operatorname{det} T_{\phi}$ which leads to $\operatorname{det} T_{\phi}$ giving rise to a non-trivial element in $H^{1}(\mathscr{G}(\tilde{\mathscr{F}}), \mathbb{Z})$.

By (3.8), $T_{\phi}=\left(\not_{b}^{-}\right)_{A} \Xi_{A, \phi}^{+}+\left(D_{b}^{-}\right)_{A}\left(D_{b}^{+}\right)_{A}$ and hence $T_{\phi}$ is a (non-symmetric) transversally elliptic operator on $\Gamma_{b}\left(E^{+}\right)$having the same symbol as $\left(\not_{b}^{-}\right)_{A}\left(\not_{b}^{+}\right)_{A}$. Outside a positive cone in $\mathbb{C}$, there are only finitely many non-zero eigenvalues $\lambda_{1}, \ldots, \lambda_{k}$. Let $\mathbf{I}-\mathbf{P}$ denote the orthogonal projection onto the finite dimensional vector space spanned by eigenfunctions corresponding to the eigenvalues $\lambda_{1}, \ldots, \lambda_{k}$, plus those in $\operatorname{ker}\left(\left(D_{b}^{+}\right)_{\phi \cdot A}\right.$ ) (in the case where $\operatorname{Ind}\left(\left(D_{b}^{+}\right)_{A}\right) \neq 0$ ). Observing that $\operatorname{ker}\left(\left(D_{b}^{+}\right)_{\phi \cdot A}\right)=\left(\hat{\phi}^{+}\right)^{-1}\left(\operatorname{ker}\left(\left(D_{b}^{+}\right)_{\phi \cdot A}\right)\right)$, one may then define

$$
\log \operatorname{det}\left(P \circ T_{\phi}\right)=\left.\frac{d}{d s}\right|_{s=0} \operatorname{Tr}\left(\left(P \circ T_{\phi}\right)^{-s}\right),
$$

by using a cut along the negative real axis in $\mathbb{C}$. In the general case, the definition

$$
\operatorname{det} T_{\phi}=\exp \left(\log \operatorname{det}\left(P \circ T_{\phi}\right)\right) \cdot \prod_{j} \lambda_{j}
$$

gives a non-vanishing determinant. The assignment $\phi \rightarrow \operatorname{det} T_{\phi}$ determines a smooth, complex valued function on $\mathscr{G}(\tilde{\mathscr{F}})$ and we define DET: $\mathscr{G}(\tilde{\mathscr{F}}) \rightarrow \mathbb{C}^{*}$ by

$$
\operatorname{DET}(\phi)=\operatorname{det} T_{\phi}=\operatorname{det}\left\{\left(\not D_{b}^{-}\right)_{A}\left(\hat{\phi}^{-}\right)^{-1}\left(\not_{b}^{+}\right)_{A} \hat{\phi}^{+}\right\} .
$$

An element of $H^{1}(\mathscr{G}(\tilde{\mathscr{F}}), \mathbb{Z})$ is now obtained by pulling back the generator $(2 \pi i)^{-1} \mathrm{~d} z / z$ of $H^{1}\left(\mathbb{C}^{*}, \mathbb{Z}\right)$ by the DET map. The image of the latter in $H^{1}(\mathscr{G}(\widetilde{\mathscr{F}})$, $\mathbb{R})$ can be represented by the differential form

$$
\omega=(2 \pi i)^{-1} d_{\mathscr{G}} \mathrm{DET} / \mathrm{DET} .
$$

Let $f \in \operatorname{Lie}(\mathscr{G}(\tilde{\mathscr{F}}))$, i.e. $f$ is an infinitesimal gauge transformation. Then the closed form $\omega_{A}$ can be expressed, for $\left(\not_{b}^{+}\right)_{A}$ invertible, by

$$
\begin{aligned}
\omega_{A}(f) & =\left.\operatorname{Tr}\left\{\left(T_{\phi}\right)^{-s}\left(\not_{b}^{+}\right)_{\phi \cdot A}^{-1}\left[\left(\not_{b}^{+}\right)_{\phi \cdot A}, \hat{f}\right]\right\}\right|_{s=0} \\
& =\operatorname{Tr}\left\{\left(D_{b}^{+}\right)_{\phi \cdot A}^{-1} \delta\left(\left(\not_{b}^{+}\right)_{\phi \cdot A}\right) / \delta f\right\} .
\end{aligned}
$$

On the other hand, for any $A \in \mathscr{A}_{b}$ and $\left(\not_{b}^{-}\right)_{A}$ non-singular, we define

$$
\tilde{\omega}_{A}(f)=\frac{\mathrm{d}}{\mathrm{d} t} \operatorname{det}\left\{\left(D_{b}^{-}\right)_{A}\left(D_{b}^{+}\right)_{e^{t f} A}\right\} /\left.\operatorname{det}\left\{\left(D_{b}^{-}\right)_{A}\left(D_{b}^{+}\right)_{e^{t f} A}\right\}\right|_{t=0} .
$$


The above form in (8.20) is not closed, but is invariant and the restriction of $\tilde{\omega}_{A}$ to an orbit and hence to $\mathscr{G}(\tilde{\mathscr{F}})$, agrees with $\omega_{A}(f)$ (closed), at the identity and gives a 1 -form $t_{1}$ on $\mathscr{G}(\tilde{\mathscr{F}})$. It can be shown that

$$
t_{1}=(2 \pi i)^{-1} d\left(\operatorname{det} T_{\phi}\right) / \operatorname{det} T_{\phi}+d f=\omega+d f,
$$

i.e.

$$
\left[t_{1}\right]=[\omega] \in H^{1}(\mathscr{G}(\tilde{\mathscr{F}}), \mathbb{R}) .
$$

We summarize this in the following theorem, using Theorem 6.1.

\section{Theorem 8.1.}

(i) The cohomology class $\mathrm{DET}^{*}\left((2 \pi i)^{-1}(\mathrm{~d} z / z)\right.$ in $H^{1}(\mathscr{G}(\tilde{\mathscr{F}}), \mathbb{Z})$ transgresses to the class $c_{1}\left(\operatorname{Ind}_{a}\left(\tilde{D}_{b}^{+}\right)\right)=\Psi_{(P, \tilde{\mathscr{F}})}^{*}\left(\tilde{c}_{1}\right) \in H^{2}(B \mathscr{G}(\tilde{\mathscr{F}}), \mathbb{Z})$.

(ii) In $H^{1}(\mathscr{G}(\widetilde{\mathscr{F}}), \mathbb{R})$, the cohomology class $\left[\mathrm{DET}^{*}\left((2 \pi i)^{-1} \mathrm{~d} z / z\right)\right]=[\omega]$ coincides with the class $\left[t_{1}\right]$ defined above.

The study of the cohomology classes in $H^{\mathrm{ev}}(B \mathscr{G}(\tilde{\mathscr{F}}), \mathbb{Z})$, in Theorem 6.1 and their suspensions in $H^{\text {odd }}(\mathscr{G}(\widetilde{\mathscr{F}}), \mathbb{Z})$ together with certain characteristic classes associated to $\mathscr{F}$, will be discussed in a separate paper (cf. e.g. [G1-K2]). In concluding, we remark that for the basic analytic families index we have

$$
c_{1}\left(\text { Ind } \tilde{D}_{b}^{+}\right)=c_{1}\left(\operatorname{det} \text { Ind } \tilde{D}_{b}^{+}\right)=c_{1}(\mathscr{L}),
$$

where $\mathscr{L}=\operatorname{det}\left(\operatorname{ker} \tilde{D}_{b}^{+}\right)^{*} \otimes \operatorname{det}\left(\operatorname{ker} \tilde{D}_{b}^{-}\right)$is the determinant line bundle defined by $\widetilde{D}_{b}^{ \pm}$. In the case where $M$ is Hermitian, a generalized Quillen metric can be constructed on $\mathscr{L}[\mathrm{Gl}-\mathrm{K} 1]$ following along the lines of [B-G-S], [F-2], [Q]. Again, does the difference $\operatorname{ch}\left(\operatorname{Ind}_{a} \widetilde{D}_{b}^{+}\right)-\operatorname{ch}\left(\operatorname{Ind}_{\text {top }} \widetilde{D}_{b}^{+}\right)$have any interpretation? In the absolute case, this is zero as a result of the families index theorem.

Acknowledgements. The authors would like to thank K. Andrew, R. Schrader and M. Stone for helpful discussions and the referee for useful comments and suggestions.

\section{References}

[A] Atiyah, M. F.: Elliptic operators and compact groups. Lecture Notes in Mathematics, Vol. 401. Berlin, Heidelberg, New York: Springer 1974

[A2] Atiyah, M. F.: Eigenvalues of the Dirac operator. Lecture Notes in Mathematics, Vol. 1111, pp. 251-260. Berlin, Heidelberg, New York: Springer 1985

[A-B1] Atiyah, M. F., Bott, R.: The Yang-Mills equations over Riemann surfaces. Phil. Trans. R. Soc. London A308, 523-616 (1982)

[A-B2] Atiyah, M. F., Bott, R.: The moment map and equivariant cohomology. Topology 23, (1) 1-28 (1984)

[A-B-P] Atiyah, M. F., Bott, R., Patodi, V. K.: On the heat equation and the index theorem. Inv. Math. 19, 279-330 (1973)

[A-H] Atiyah, M. F., Hitchin, N. J.: The geometry and dynamics of magnetic monopoles. Princeton, NJ: Princeton Univ. Press. 1988

[A-J] Atiyah, M. F., Jones, J. D. S.: Topological Aspects of Yang-Mills Theory. Commun. Maths. Phys. 61, 97-118 (1978)

[A-P-S] Atiyah, M. F., Patodi, V. K., Singer, I. M.: Spectral asymmetry and Riemannian geometry. Math. Proc. Camb. Phil. Soc., I: 77, 43-69 (1975); II: 78, 405-432 (1976); III: 79, 71-99 (1976) 
[A-S1] Atiyah, M. F., Singer, I. M.: The index of elliptic operators III. Ann. Math. 87, 546-604 (1968)

[A-S2] Atiyah, M. F., Singer, I. M.: The index of elliptic operators IV. Ann. Math. 93, 119-138 (1971)

[A-S3] Atiyah, M. F., Singer, I. M.: Index theory for skew-adjoint Fredholm operators. Publ. Math. I.H.E.S. 39, 305-326 (1969)

[A-S4] Atiyah, M. F., Singer, I. M.: Dirac operators coupled to vector potentials. Proc. Nat1. Acad. Sci. USA 81, 2597-2600 (1984)

[B-G-S] Bismut, J. H., Gillet, H., Soulé, C.: Analytic torsion and holomorphic determinant line bundles III. Commun. Math. Phys. 115, 301-351 (1988)

[B-C-R-S] Bonora, L., Cotta-Ramusino, P., Rinaldi, M., Stasheff, J.: The evaluation map in field theory, sigma models and strings. Commun. Math. Phys. I: 112, 237-282 (1987); II: 114, 381-437 (1988)

[Bo] Bott, R.: Lectures on characteristic classes and foliations. Lecture Notes in Math. Vol. 279, pp. 1-94. Berlin, Heidelberg, New York: Springer 1972

[Br] Brüning, J.: Spectral analysis on singular spaces. Preprint, University of Augsburg (1989)

[B-H1] Brüning, J., Heintze, E.: Representations of compact Lie groups and elliptic operators. Inv. Math. 50, 169-203 (1979)

[B-H2] Brüning, J., Heintze, E.: The asymptotic expansion of Minakshisundaram-Pleyel in the equivariant case, Duke Math. J. 51, 959-980 (1984)

[B-K1] Brüning, J., Kamber, F. W.: Vanishing theorems and index formulas for transversal Dirac operators. A.M.S. Meeting 845, Special Session on Operator Theory and Applications to Geometry, Lawrence, KA; A.M.S. Abstracts, October 1988

[B-K2] Brüning, J., Kamber, F. W.: On the spectrum and index of transversal Dirac operators associated to Riemannian foliations (to appear)

[B-Sc] Brüning, J., Schröder, H.: On the absence of log terms in the constant curvature case. Asympt. Anal. 1, 193-203 (1988)

[Co] Connes, A.: Non-commutative differential geometry. Publ. Math. IHES 62, 41-144 (1985)

[EK] ElKacimi, A.: Opérateurs transversalements elliptiques. Publ. IRMA-Lille 7, III/1-44 (1986)

[EK-H] ElKacimi, A., Hector, G.: Décomposition de Hodge basique pour un feuilletage Riemannien. Ann. Inst. Fourier 36, 207-227 (1986)

[F-1] Freed, D. S.: An index theorem for families of Fredholm operators parametrized by a group. Topology 27, (3) 279-300 (1988)

[F-2] Freed, D. S.: On determinant line bundles, in Mathematical Aspects of String Theory. Singapore: World Scientific 1989

[F-V] Freed, D. S., Vafa, C.: Global anomalies on orbifolds. Commun. Math. Phys. 110, 349-389 (1987)

[Gi] Gilkey, P.: Invariance theory, the heat equation and the Atiyah-Singer index theorem. Math. Lect. Series. Vol. 11, Publ. or Perish, 1984

[Gl-K1] Glazebrook, J. F., Kamber, F. W.: Determinant line bundles for Hermitian foliations and a generalized Quillen metric. AMS Symp. Pure Math. "Several Complex Variables," Santa Cruz 1989 (to appear)

[G1-K2] Glazebrook, J. F. Kamber, F. W.: Chiral anomalies and Dirac families in Riemannian foliations (to appear)

[G-L1] Gromov, M., Lawson, H. B.: Spin and scalar curvature in the presence of a fundamental group I. Ann. Math. 111, 209-230 (1980)

[G-L2] Gromov, M., Lawson, H. B.: Positive scalar curvature and the Dirac operator on complete Riemannian manifolds. Publ. Math. I.H.E.S. 58, 83-196 (1983)

[J-T] Jaffe, A., Taubes, C.: Vortices and monopoles. Boston, M. A.: Birkhäuser 1980

[K-T1] Kamber, F. W., Tondeur, Ph.: Foliated bundles and characteristic classes. Lect. Notes in Math. Vol. 493, Berlin, Heidelberg, New York: Springer 1975

[K-T2] Kamber, F. W., Tondeur, Ph.: Harmonic foliations. Lecture Notes in Mathematics Vol. 949, 87-121 Berlin, Heidelberg, New York: Springer 1982

[K-T3] Kamber, F. W., Tondeur, Ph.: Duality for Riemannian foliations. Proc. Symp. Pure Math. 40 (1) 609-618 (1983) 
[K-T4] Kamber, F. W., Tondeur, Ph.: Foliations and metrics. Proc. of a Year in Differential Geometry, Univ. of Maryland, Progr. Math., Vol. 32, 103-152. Boston, M. A.: Birkhäuser 1983

[K-T5] Kamber, F. W., Tondeur, Ph.: DeRham-Hodge theory for Riemannian foliations. Math. Ann. 277, 415-431 (1987)

[Li] Lichnerowicz, A.: Spineurs harmoniques. C.R.A.S., Paris, Sér. A, 257, 7-9 (1963)

[M-R] Mickelsson, J., Rajeev, S. G.: Current algebras in $(d+1)$-dimensions and determinant bundles over infinite dimensional Grassmannians. Commun. in Math. Phys. 116, $365-400$

[Mo] Molino, P.: Riemannian foliations. Progr. Math., Vol. 73. Boston, M. A.: Birkhäuser 1988

[P] Palais, R. S.: On the homotopy type of certain groups of operators. Topology 3, 271-279 (1965)

[Q] Quillen, D.: Determinants of Cauchy-Riemann operators on a Riemann surface. Funk. Anal. i ego Priloz. 19, 37-41 (1985)

[P-S] Pressley, A. N., Segal, G. B.: Loop groups and their representations. Oxford: Oxford Univ. Press. 1986

[Re1] Reinhart, B.: Foliated manifolds with bundle-like metrics. Ann. Math. 69, 119-132 (1959)

[Re2] Reinhart, B.: The differential geometry of foliations. Erg. Math. Vol. 99. Berlin, Heidelberg, New York: Springer 1983

[Ro1] Roe, J.: Analysis on manifolds. D. Phil. Thesis, Oxford 1984

[Ro2] Roe, J.: Finite propagation speed and Connes' foliation algebra. Math. Proc. Camb. Phil. Soc. 102, 459-466 (1987)

[Ro3] Roe, J.: An index theorem on open manifolds I/II. J. Diff. Geom. I: 27, 87-113-136 (1988)

[Ru] Rummler, H.: Quelques notions simples en géométrie riemannienne et leurs applications aux feuilletages compacts. Comm. Math. Helv. 54, 224-239 (1979)

[Se] Seeley, R. T.: Complex powers of elliptic operators. Proc. Symp. Pure Math. 10, 288-307 (1967)

[Si] Singer, I. M.: Families of Dirac operators with applications to physics. Astérisque (hors série) 323-340 (1985)

[V-W] Vafa, C., Witten, E.: Eigenvalue inequalities for fermions in gauge theories. Commun. Math. Physics 95 (3), 257-276 (1984)

[W] Witten, E.: A new proof of the positive energy theorem. Commun. Math. Phys. 80, 381-402 (1981)

Communicated by A. Jaffe 